\title{
IN-PLANE NONLINEAR ANALYSIS AND BUCKLING OF SHEAR-DEFORMABLE CIRCULAR ARCHES
}

\author{
Gen-shu Tong ${ }^{1, *}$, Yong-lin $\mathrm{Pi}^{2}$ and Wei Gao ${ }^{2}$ \\ ${ }^{1}$ Department of Civil Engineering, Zhejiang University, Hangzhou 310058, China, \\ ${ }^{2}$ Department of Civil \& Environmental Engineering, The University of New South Wales, NSW 2052, Australia \\ *( corresponding author, tonggs@zju.edu.cn)
}

\section{A B S T R A C T}

A new theory for nonlinear analysis of shear-deformable circular arches is derived, in which Timoshenko's assumption on the deformation of cross-section and the Green strains are adopted. In the variational equation, the nonlinear energy of the shear and the transverse normal stresses is included. Substituting the internal forces from linear analysis, a set of linearized equations is derived for buckling analysis of shear deformable arches. These equations are then used to solve the buckling of circular arches and rings under three types of radial pressures to compare the various results appeared in the literature.

Linear analysis is carried out on hinged arches under uniform radial pressure to check the changes of displacements and internal forces after the shear deformation is considered. It is found that the axial force is more uniform along the arch length when shear deformation is considered, and the bending moment and shear force are smaller, but the displacements are always larger.

Buckling of arches under radial pressure under various boundary conditions are studied, buckling factors for symmetrical and anti-symmetrical buckling are tabulated, and approximate formulas for the critical loads are proposed.
ART I CLE H I S T O R Y

$\begin{array}{ll}\text { Received: } & \text { 22 February } 2019 \\ \text { Revised: } & \text { 15 January } 2020 \\ \text { Accepted: } & \text { 20 January } 2020\end{array}$

\section{K E Y W O R D S}

Circular arch;

Buckling;

Nonlinear analysis;

Shear deformation;

Linear analysis

Copyright $(2020$ by The Hong Kong Institute of Steel Construction. All rights reserved.

\section{Introduction}

In-plane buckling of arches is a classic problem $[1,2,3]$. The early literature review on arches with Bernoulli's assumption was presented in Ref. [4]. This paper will address the buckling of circular arches whose shear deformation cannot be neglected. Such a situation occurs when the arch is a trussed arch, or the web of the arch has openings with regular intervals. Early studies on shear-deformable arches were mainly focused on their vibration $[5,6]$.

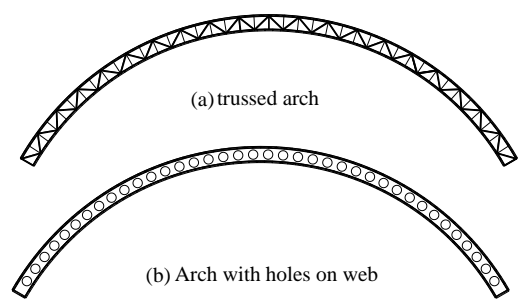

Fig.1 Shear deformable arches

Study on buckling of arches incorporating shear deformation of their cross-sections may date back to the early Russian literature [7]. Dinnik [7], without any introduction to the theoretical development, presented Eq. (1) as the critical force of hinged arches under water pressure:

$(q R)_{\mathrm{cr}}=\frac{1}{1+\Phi_{s}}\left(1-\frac{\alpha^{2}}{\pi^{2}}\right) \frac{\pi^{2} E I}{(\alpha R)^{2}}, \quad \Phi_{s}=\frac{\pi^{2}}{\alpha^{2}} \Phi, \quad \Phi=\frac{E I}{G A_{s} R^{2}}$

Where $E, G$ are Young's and shear moduli of steel respectively, $I$ the second moment of the cross-section, $A_{s}$ the effective shear area, $R$ the centroidal radius of the arch, $\alpha$ half of the subtended angle of the arch. Based on Ref. [7], Eq. (1) was obtained by Bovin in 1937, but no further information is available because of the language limitation and the long history.

Smith and Simitses [8] investigated also the effect of transverse shear deformation on buckling of rings and developed the following equation for the fundamental mode of buckling $(n=1)$ :

$$
p R=\frac{1}{1+4 \Phi} \cdot \frac{3 E I}{R^{2}}
$$

Guo and Huang [9], Dou et al. [10] studied the buckling of arches with I-section with regular web openings numerically, and used the following equation to define the slenderness of arches. $(q R)_{\mathrm{cr}}=\frac{1}{1+\left(1-\alpha^{2} / \pi^{2}\right) \Phi_{s}}\left(1-\frac{\alpha^{2}}{\pi^{2}}\right) \frac{\pi^{2} E I}{(\alpha R)^{2}}$

Guo et al. [11] investigated the buckling and load-carrying capacity of 3D-trussed arches, Guo et al. [12] studied the arches with corrugated webs. Attard, Zhu and Kellermann [13] carried out a new study on the effect of shear deformation, using finite strain formulation, the following critical load was obtained:

$(p R)_{\mathrm{cr}}=\left[\frac{1}{2}\left(1+\frac{1}{\Phi}\right)+\sqrt{\frac{1}{4}\left(1+\frac{1}{\Phi}\right)^{2}+\left(\frac{\pi^{2}}{\alpha^{2}}-1\right) \frac{1}{\Phi}}\right] \frac{E I}{R^{2}}$

Zhu, Attard and Kellermann [14] investigated the nonlinear buckling of shallow shear-deformable arches, Attard, Zhu and Kellermann [15] studied the buckling of funicular (parabolic and catenary) shear-deformable arches.

There exist great differences among Eqs. $(1,3,4)$. This paper presents a new study on the buckling of shear-deformable circular rings and arches under radial pressure. The theoretical derivation is an extension of the authors' early study on arches based on Bernoulli's assumption [4].

\section{A nonlinear theory of arches incorporating shear deformation}

\subsection{Assumptions and coordinate systems}

Fig.2 illustrates the following right-handed coordinate systems:

(1) $r-\varphi-y$ is a cylindrical coordinate system located at the curvature center of the arch, it is fixed in space and used to measure the deformations of the arch;

(2) $x-y-z$ is a Cartesian coordinate system located at the centroid of the cross-section, it is fixed on the cross-section and changes its direction as the arch deforms. The original $x$-axis has the same direction as the $r$-axis, the $z$-axis points in the direction of increasing $\varphi$.

The cross-section of the arch is bi-symmetrical, the radius of the centroidal axis is $R$. The displacements of any point $\mathrm{P}(x, y)$ on the cross-section are denoted by $\bar{u}$ and $\bar{w}$ in the $x$ - and $z$-directions, respectively. Following assumptions are adopted in the derivation:

(2) The cross section has dimensions far less than the radius of the arch;

(2) $(2)$ The cross-section remains plane but not perpendicular to the deformed axis;

(3) The displacements may be finite, but the strain is small. 


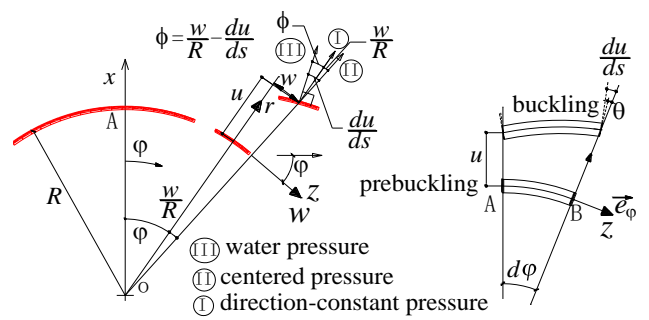

Fig.2 Coordinate systems, deformation of the cross-section

\subsection{The strain-displacement relations}

Referring to Fig.2, $\bar{u}$ and $\bar{w}$ of Point $\mathrm{P}(x, y)$ on the middle surface of the cross-section in the $x$ and $z$ directions can be written out

$\bar{u}=u, \quad \bar{w}=\frac{r}{R} w-x \theta$

in which $u$ and $w$ are the displacements of the centroid of the cross section in the $x$ and $z$ directions respectively, $\theta$ is the rotation of the cross-section due to pure flexural deformation. ()$^{\prime}=\partial() / \partial \varphi, r$ is the radius of Point $\mathrm{P}(x, y)$. There are three independent strain components, each of them is composed of linear and nonlinear parts.

$\varepsilon_{z}=\varepsilon_{z}^{\mathrm{L}}+\varepsilon_{z}^{\mathrm{N}}, \quad \varepsilon_{z x}=\varepsilon_{z x}^{\mathrm{L}}+\varepsilon_{z x}^{\mathrm{N}}, \quad \varepsilon_{x}=\varepsilon_{x}^{\mathrm{L}}+\varepsilon_{x}^{\mathrm{N}}$

$(6 a, b, c)$

Strain-displacement relations are expressed as [16]:

$\varepsilon_{\mathrm{x}}^{\mathrm{L}}=\frac{\partial \bar{u}}{\partial x}=\frac{\partial u}{\partial x}=0 \quad(\mathrm{web})$

$\varepsilon_{\mathrm{z}}^{\mathrm{L}}=\frac{\partial \bar{w}}{r \partial \phi}+\frac{\bar{u}}{r}=\frac{u}{r}+\frac{1}{R} w^{\prime}-\frac{x}{r} \theta^{\prime}$

$\varepsilon_{\mathrm{xz}}^{\mathrm{L}}=\frac{\partial \bar{u}}{r \partial \varphi}-\frac{\bar{w}}{r}+\frac{\partial \bar{w}}{\partial x}=\frac{u^{\prime}-R \theta}{r}=\frac{R}{r}\left(\frac{u^{\prime}}{R}-\theta\right)$

$\varepsilon_{\mathrm{x}}^{\mathrm{N}}=\frac{1}{2}\left[\left(\frac{\partial \bar{u}}{\partial x}\right)^{2}+\left(\frac{\partial \bar{w}}{\partial x}\right)^{2}\right]=\frac{1}{2}\left(\frac{\partial \bar{w}}{\partial x}\right)^{2}$

$\varepsilon_{\mathrm{z}}^{\mathrm{N}}=\frac{1}{2}\left[\left(\frac{\partial \bar{u}}{r \partial \phi}-\frac{\bar{w}}{r}\right)^{2}+\left(\frac{\partial \bar{w}}{r \partial \phi}+\frac{\bar{u}}{r}\right)^{2}\right]$

$\varepsilon_{\mathrm{zx}}^{\mathrm{N}}=\frac{\partial \bar{u}}{\partial x}\left(\frac{\partial \bar{u}}{r \partial \phi}-\frac{\bar{w}}{r}\right)+\frac{\partial \bar{w}}{\partial x}\left(\frac{\partial \bar{w}}{r \partial \phi}+\frac{\bar{u}}{r}\right)=\frac{\partial \bar{w}}{\partial x}\left(\frac{\partial \bar{w}}{r \partial \phi}+\frac{u}{r}\right)$

$\varepsilon_{\mathrm{y}}^{\mathrm{L}}=0, \varepsilon_{\mathrm{y}}^{\mathrm{N}}=\frac{1}{2}\left(\frac{\partial u}{\partial y}\right)^{2}+\frac{1}{2}\left(\frac{\partial v}{\partial y}\right)^{2}+\frac{1}{2}\left(\frac{\partial \bar{w}}{\partial y}\right)^{2}=0$ (flanges)

Substituting Eqs. (5a, 5b) into Eqs. (7a-5f), one obtains

$\varepsilon_{\mathrm{z}}^{\mathrm{L}}=\varepsilon_{\mathrm{m}}-\frac{x}{r} \kappa, \quad \varepsilon_{\mathrm{z}}^{\mathrm{N}}=\frac{1}{2}\left[\left(\frac{R}{r} \gamma+\beta\right)^{2}+\left(\varepsilon_{\mathrm{m}}-\frac{x}{r} \kappa\right)^{2}\right]$

$\varepsilon_{\mathrm{x}}^{\mathrm{L}}=0, \quad \varepsilon_{\mathrm{x}}^{\mathrm{N}}=\frac{1}{2} \beta^{2}($ web $)$

$\varepsilon_{\mathrm{xz}}^{\mathrm{L}}=\frac{R}{r} \gamma, \quad \varepsilon_{\mathrm{zx}}^{\mathrm{N}}=-\beta\left(\varepsilon_{\mathrm{m}}-\frac{x}{r} \kappa\right)$

Where

$\varepsilon_{\mathrm{m}}=\frac{w^{\prime}+u}{R}, \quad \kappa=\frac{u}{R}+\theta^{\prime}, \quad \beta=\theta-\frac{w}{R}, \quad \gamma=\frac{u^{\prime}}{R}-\theta$

$(9 \mathrm{a}, \mathrm{b}, \mathrm{c}, \mathrm{d})$

$\gamma$ is the shear angle of the cross-section. There are following relations between these quantities $\varepsilon_{m}-\kappa=-\beta^{\prime}, \quad \beta+\gamma=\frac{u^{\prime}-w}{R}, \quad \kappa+\gamma^{\prime}=\frac{u+u^{\prime \prime}}{R}$

$(10 \mathrm{a}, \mathrm{b}, \mathrm{c})$

Stresses on the cross-sections are $\sigma_{\mathrm{z}}, \tau_{\mathrm{xz}}$ and $\sigma_{\mathrm{x}}$ (in the web) and $\sigma_{\mathrm{y}}$ (in the flanges). The external loads are balanced by the longitudinal normal stress $\sigma_{z}$ and the shear stress $\tau_{\mathrm{xz}}$, the transverse normal stress $\sigma_{\mathrm{x}}\left(\sigma_{\mathrm{y}}\right)$ are produced to keep equilibrium of the infinitesimal sectorial plate elements(web) or cylindrical elements(flange). $\sigma_{z}$ is related to the normal strain, $\tau_{x z}$ is found based on the equilibrium of infinitesimal elements, it is also related to the shear deformation in an averaged sense because Timoshenko's assumption is only concerned with cross-sectional average shear deformation. $\sigma_{\mathrm{x}}\left(\sigma_{\mathrm{y}}\right)$ can only be determined by equilibrium condition of the infinitesimal element.

Based on Hooke's law, the stresses $\sigma_{\mathrm{z}}$ and $\tau_{\mathrm{zx}}$ are

$\sigma_{\mathrm{z}}=E\left(\varepsilon_{\mathrm{z}}^{\mathrm{L}}+\varepsilon_{\mathrm{z}}^{\mathrm{N}}\right)=E\left\{\varepsilon_{\mathrm{m}}-\frac{x}{r} \kappa+\frac{1}{2}\left[\left(\frac{R}{r} \gamma+\beta\right)^{2}+\left(\varepsilon_{\mathrm{m}}-\frac{x}{r} \kappa\right)^{2}\right]\right\}$

$\tau_{\mathrm{xz}}=G\left(\varepsilon_{\mathrm{xz}}^{\mathrm{L}}+\varepsilon_{\mathrm{xz}}^{\mathrm{N}}\right)=E\left[\frac{R}{r} \gamma-\beta\left(\varepsilon_{\mathrm{m}}-\frac{x}{r} \kappa\right)\right]$

The axial force $N$, bending moment $M$ and shear force $Q_{\mathrm{x}}$ on the cross section are:

$N=\int_{A} \sigma_{\mathrm{z}} \mathrm{d} A=E \int_{A}\left(\varepsilon_{\mathrm{m}}-\frac{x}{r} \kappa\right) \mathrm{d} A=E A \varepsilon_{\mathrm{m}}+\frac{E I}{R^{2}} \kappa$

$M=-\int_{A} \sigma_{\mathrm{z}} x \mathrm{~d} A=-E \int_{A}\left(\varepsilon_{\mathrm{m}}-\frac{x}{r} \kappa\right) x \mathrm{~d} A=\frac{E I}{R} \kappa$

$Q_{\mathrm{x}}=\int_{A} \tau_{\mathrm{zx}} \mathrm{d} A=G R \gamma \int_{A} \frac{1}{r} t_{w} d r=G t_{w} R \gamma \ln \frac{R+0.5 h}{R-0.5 h} \approx G A_{\mathrm{s}} \gamma$

The axial force is positive when it is tensile, the bending moment is positive when the inner side of the arch is in tension, the shear force is positive when it points in the positive $x$ direction on the positive cross-section.

2.2 Principle of virtual work and equilibrium equation

For arches, the virtual work equation is

$\int_{V}\left(\sigma_{\mathrm{z}} \delta \varepsilon_{\mathrm{z}}^{\mathrm{L}}+\tau_{\mathrm{zx}} \delta \varepsilon_{\mathrm{zx}}^{\mathrm{L}}+\sigma_{\mathrm{z}} \delta \varepsilon_{\mathrm{z}}^{\mathrm{N}}+\tau_{\mathrm{zx}} \delta \varepsilon_{\mathrm{zx}}^{\mathrm{N}}+\sigma_{\mathrm{x}} \delta \varepsilon_{\mathrm{x}}^{\mathrm{N}}\right) \mathrm{d} V=\int_{\varphi}\left(q_{\mathrm{z}} \delta w+q_{\mathrm{x}} \delta u\right) R \mathrm{~d} \varphi($

where $\mathrm{d} V=r \mathrm{~d} A \mathrm{~d} \varphi$ and

$\delta \varepsilon_{\mathrm{z}}^{\mathrm{L}}=\delta \varepsilon_{m}-\frac{x}{r} \delta \kappa, \delta \varepsilon_{x}^{\mathrm{N}}=\beta \delta \beta$

$\delta \varepsilon_{\mathrm{z}}^{\mathrm{N}}=\left(\frac{R}{r} \gamma+\beta\right)\left(\frac{R}{r} \delta \gamma+\delta \beta\right)+\left(\varepsilon_{\mathrm{m}}-\frac{x}{r} \kappa\right)\left(\delta \varepsilon_{\mathrm{m}}-\frac{x}{r} \delta \kappa\right)$

$\delta \varepsilon_{\mathrm{xz}}^{\mathrm{L}}=\frac{R}{r} \delta \gamma, \delta \varepsilon_{\mathrm{zx}}^{\mathrm{N}}=-\delta \beta\left(\varepsilon_{\mathrm{m}}-\frac{x}{r} \kappa\right)-\beta\left(\delta \varepsilon_{\mathrm{m}}-\frac{x}{r} \delta \kappa\right)$

$\delta \varepsilon_{\mathrm{m}}=\frac{\delta w^{\prime}+\delta u}{R}, \quad \delta \kappa=\frac{\delta u}{R}+\delta \theta^{\prime}, \quad \delta \beta=\delta \theta-\frac{\delta w}{R}, \quad \delta \gamma=\frac{\delta u^{\prime}}{R}-\delta \theta$

(1) The first part of the virtual work is related to the linear longitudinal strain:

$\int_{V} \sigma_{\mathrm{z}} \delta \varepsilon_{\mathrm{z}}^{\mathrm{L}} \mathrm{d} V=\int_{\varphi} \int_{A} \sigma_{\mathrm{z}}\left(\delta \varepsilon_{\mathrm{m}}-\frac{x}{r} \delta \kappa\right) r \mathrm{~d} A \mathrm{~d} \varphi=\int\left[(R N-M) \delta \varepsilon_{\mathrm{m}}+M \delta \kappa\right] \mathrm{d} \varphi(16 \mathrm{a})$

in which

$\int_{A} \sigma_{z} r \mathrm{~d} A=R N-M, \quad N=\int_{A} \sigma_{z} d A, \quad M=-\int_{A} \sigma_{z} x d A$

$(16 b, c, d)$

(2) The second part is related to the nonlinear longitudinal strain: 
$\int_{V} \sigma_{\mathrm{z}} \delta \varepsilon_{\mathrm{z}}^{\mathrm{N}} \mathrm{d} V=\int_{\varphi}[(R N-M+W) \cdot \gamma \delta \gamma+N R \beta \delta \gamma+N R \gamma \delta \beta] \mathrm{d} \varphi$

$+\int_{\varphi}\left[(R N-M) \beta \delta \beta+(R N-M) \varepsilon_{\mathrm{m}} \delta \varepsilon_{\mathrm{m}}+M \varepsilon_{\mathrm{m}} \delta \kappa+M \kappa \delta \varepsilon_{\mathrm{m}}+W \kappa \delta \kappa\right] \mathrm{d} \varphi$

(17)

Where

$\int_{A} \sigma_{\mathrm{z}} \frac{R^{2}}{r} \mathrm{~d} A=R N-M+W, \quad W=\int_{A} \sigma_{\mathrm{z}} \frac{x^{2}}{r} \mathrm{~d} A$

(3) The linear part of the virtual work of the shear stress $\tau_{\mathrm{zx}}$ is

$\int_{V} \tau_{\mathrm{zx}} \delta \varepsilon_{z \mathrm{x}}^{\mathrm{L}} \mathrm{d} V=\int_{\varphi} \int_{A} \tau_{z \mathrm{x}} \frac{R}{r} \delta \gamma r \mathrm{~d} A \mathrm{~d} \varphi=R \int_{\varphi} \int_{A} \tau_{\mathrm{zx}} \delta \gamma \mathrm{d} A \mathrm{~d} \varphi=R \int_{\varphi} Q_{\mathrm{x}} \delta \gamma \mathrm{d} \varphi$

The nonlinear part of the virtual work of the shear stress $\tau_{\mathrm{zx}}$

$\int_{V} \tau_{\mathrm{zx}} \delta \varepsilon_{\mathrm{zx}}^{\mathrm{N}} \mathrm{d} V=\int_{\varphi}\left[\left(R Q_{\mathrm{x}}+T\right)\left(-\beta \delta \varepsilon_{\mathrm{m}}-\varepsilon_{\mathrm{m}} \delta \beta\right)+T(\kappa \delta \beta+\beta \delta \kappa)\right] \mathrm{d} \varphi$

where

$\int_{A} \tau_{\mathrm{zx}} r \mathrm{~d} A=R Q_{\mathrm{x}}+T, \quad T=\int_{A} \tau_{\mathrm{zx}} x \mathrm{~d} A$

(4) the nonlinear virtual work of the transverse normal stress $\sigma_{\mathrm{x}}$ is

$\int_{V} \sigma_{\mathrm{x}} \delta \varepsilon_{\mathrm{x}}^{\mathrm{N}} \mathrm{d} V=\int_{\varphi} \int_{A} \sigma_{\mathrm{x}} \beta \delta \beta r \mathrm{~d} A \mathrm{~d} \varphi=\int_{\varphi}\left[\int_{A} \sigma_{\mathrm{x}} r \mathrm{~d} A\right] \beta \delta \beta \mathrm{d} \varphi$

$$
\begin{gathered}
\sigma_{x}+\tau_{x z}+d \tau_{x z} \\
\sigma_{x} \tau_{\tau_{z x}} \frac{\tau_{z x}+d \tau_{z x}}{\sigma_{\sigma_{x}}} \sigma_{z}+d \sigma_{z} \\
\sigma^{\prime}
\end{gathered}
$$

Fig.3 Balance of infinitesimal sectorial element

Fig. 3 shows an infinitesimal element, its equilibrium equation in the radial direction is:

$\frac{\partial \sigma_{\mathrm{x}} t}{\partial x}+\frac{\partial \tau_{z x} t}{r \partial \varphi}-\frac{\sigma_{\mathrm{z}} t-\sigma_{\mathrm{x}} t}{r}=0$

from which

$\frac{\partial\left(\sigma_{\mathrm{x}} r t\right)}{\partial r}=\sigma_{\mathrm{z}} t-\frac{\partial \tau_{z x} t}{\partial \varphi}$

Multiplying both sides by $x$, integration along the height of the cross section, one has

$\int_{-0.5 h}^{0.5 h} \frac{\partial\left(\sigma_{x} r t\right)}{\partial x} x \mathrm{~d} x=\int_{-0.5 h}^{0.5 h} \sigma_{\mathrm{z}} t x \mathrm{~d} x-\int_{-0.5 h}^{0.5 h} \frac{\partial\left(\tau_{z x} t\right)}{\partial \varphi} x \mathrm{~d} x=-M-\frac{\partial T}{\partial \varphi}$

on the other hand

$\int_{-0.5 h}^{0.5 h} \frac{\partial\left(\sigma_{\mathrm{x}} r t\right)}{\partial x} x \mathrm{~d} x=\left.\sigma_{\mathrm{x}} r t \cdot x\right|_{-0.5 h} ^{0.5 h}-\int_{-0.5 h}^{0.5 h} \sigma_{\mathrm{x}} r t \mathrm{~d} x=-M-\frac{\partial T}{\partial \varphi}$

Therefore

$\int_{-0.5 h}^{0.5 h} \sigma_{\mathrm{x}} r \mathrm{~d} A=M+T^{\prime}+\left.\sigma_{\mathrm{x}} r t \cdot x\right|_{-0.5 h} ^{0.5 h}$

If the radial load $q_{x}$ is on the centroid, then $\left.\sigma_{x}\right|_{x=-0.5 h}=0,\left.\sigma_{x}\right|_{x=0.5 h}=0$, $\left.\sigma_{x}\right|_{x=-0.0}+q_{x}=\left.\sigma_{x}\right|_{x=+0.0}$, one has $\left.\sigma_{\mathrm{x}} r t \cdot x\right|_{-0.5 h} ^{0.5 h}=\left.\sigma_{\mathrm{x}} r t \cdot x\right|_{-0.5 h} ^{0}+\left.\sigma_{\mathrm{x}} r t \cdot x\right|_{0} ^{0.5 h}=0$, $\int_{A} \sigma_{\mathrm{x}} r \mathrm{~d} A=M+T^{\prime}$. Similarly, if the radial load is on the outer surface: $\int_{A} \sigma_{\mathrm{x}} r \mathrm{~d} A=M+T^{\prime}+0.5 q_{\mathrm{x}}(R+0.5 h) h$, and if the radial load is on the inner surface: $\int_{A} \sigma_{\mathrm{x}} r \mathrm{~d} A=M+T^{\prime}-0.5 q_{\mathrm{x}}(R-0.5 h) h$, so for the radial loads at any position on the cross-section, one obtains

$\int_{A} \sigma_{\mathrm{x}} r \mathrm{~d} A=M+T^{\prime}+q_{\mathrm{x}}\left(R+x_{\mathrm{q}}\right) x_{\mathrm{q}}=M+T^{\prime}+q_{\mathrm{x}} R_{\mathrm{q}} x_{\mathrm{q}}$

where $R_{q}$ is the radius on which the radial load acts, $x_{q}$ is the coordinate.

$\int_{V} \sigma_{\mathrm{x}} \delta \varepsilon_{\mathrm{x}}^{\mathrm{N}} \mathrm{d} V=\int_{\phi}\left(M+T^{\prime}+q_{\mathrm{x}} R_{\mathrm{q}} x_{\mathrm{q}}\right) \beta \delta \beta \mathrm{d} \phi$

The final virtual work equation is:

$\int_{\phi}\left[(R N-M)\left(\delta \varepsilon_{\mathrm{m}}+\beta \delta \beta+\varepsilon_{\mathrm{m}} \delta \varepsilon_{\mathrm{m}}\right)+M \delta \kappa+R Q_{\mathrm{x}} \delta \gamma+M \delta\left(\varepsilon_{\mathrm{m}} \kappa\right)\right.$

$+W \kappa \delta \kappa+\left(M+T^{\prime}+q_{\mathrm{x}} R_{\mathrm{q}} x_{\mathrm{q}}\right) \beta \delta \beta+\left(R Q_{\mathrm{x}}+T\right)\left(-\beta \delta \varepsilon_{\mathrm{m}}-\varepsilon_{\mathrm{m}} \delta \beta\right)$

$+T(\kappa \delta \beta+\beta \delta \kappa)] \mathrm{d} \varphi+\int_{\phi}[(R N-M+W) \cdot \gamma \delta \gamma+N R \beta \delta \gamma+N R \gamma \delta \beta] \mathrm{d} \varphi$

$-\int_{\phi}\left(q_{\mathrm{x}} \delta u+q_{\mathrm{z}} \delta w\right) R \mathrm{~d} \varphi=0$

Substituting Eqs. (15a-d) into Eqs. (24), and combining like terms

$\int_{\phi}\left\{\left[N-\frac{M}{R}+\left(N-\frac{M}{R}\right) \varepsilon_{\mathrm{m}}+\frac{M}{R} \kappa-\left(Q_{\mathrm{x}}+\frac{T}{R}\right) \beta\right] \delta w^{\prime}\right.$

$+\left[\left(Q_{\mathrm{x}}+\frac{T}{R}\right) \varepsilon_{\mathrm{m}}-\left(N+\frac{T^{\prime}+q_{\mathrm{x}} R_{\mathrm{q}} x_{\mathrm{q}}}{R}\right) \beta-\frac{T}{R} \kappa-N \gamma\right] \delta w$

$+\left[M+M \varepsilon_{\mathrm{m}}+W \kappa+T \beta\right] \delta \theta^{\prime}+\left[N+N \varepsilon_{\mathrm{m}}+\frac{M+W}{R} \kappa-Q_{\mathrm{x}} \beta\right] \delta u$

$+\left[\left(T^{\prime}+q_{\mathrm{x}} R_{\mathrm{q}} x_{\mathrm{q}}\right) \beta-R Q_{\mathrm{x}}+T \kappa+(M-W) \cdot \gamma-\left(R Q_{\mathrm{x}}+T\right) \varepsilon_{\mathrm{m}}\right] \delta \theta$

$\left.+\left[Q_{\mathrm{x}}+\left(N+\frac{W-M}{R}\right) \cdot \gamma+N \beta\right] \delta u^{\prime}\right\} \mathrm{d} \varphi-\int_{\phi}\left(q_{\mathrm{x}} \delta u+q_{\mathrm{z}} \delta w\right) R \mathrm{~d} \varphi=0$

Carrying out partial integration and introducing $\varepsilon_{\mathrm{m}}-\kappa=-\beta^{\prime}$ :

$\int_{-\alpha}^{\alpha}\left\{\left[-N^{\prime}+\frac{M^{\prime}}{R}-\frac{M \beta^{\prime \prime}}{R}+\left(Q_{x}^{\prime}-N-\frac{q_{\mathrm{x}} R_{\mathrm{q}} x_{\mathrm{q}}}{R}\right) \beta+\left(Q_{x}-\frac{M^{\prime}}{R}\right) \beta^{\prime}-N \varepsilon_{m}^{\prime}-N \gamma\right.\right.$

$\left.+\left(Q_{x}-N^{\prime}\right) \varepsilon_{m}-q_{z} R\right] \delta w+\left[-M^{\prime}-R Q_{x}-\left(M \varepsilon_{m}\right)^{\prime}-(W \kappa)^{\prime}-R Q_{x} \varepsilon_{m}-W \cdot \gamma\right.$

$\left.+M \gamma+q_{\mathrm{x}} R_{\mathrm{q}} x_{\mathrm{q}} \beta\right] \delta \theta+\left[N-Q_{x}^{\prime}-q_{x} R+N\left(\varepsilon_{m}-\beta^{\prime}\right)-\left(Q_{x}+N^{\prime}\right) \beta+\frac{M \kappa+W \kappa}{R}\right.$

$\left.\left.-\left[\left(N+\frac{W-M}{R}\right) \gamma\right]^{\prime}\right] \delta u\right\} d \varphi+\left.\left[N-\frac{M}{R}\left(1+\varepsilon_{m}-\kappa\right)+N \varepsilon_{m}-\left(Q_{x}+\frac{T}{R}\right) \beta\right] \delta w\right|_{-\alpha} ^{\alpha}$

$+\left.\left(M+M \varepsilon_{m}+W \kappa+T \beta\right) \frac{\delta \theta}{R}\right|_{-\alpha} ^{\alpha}+\left.\left(Q_{x}+\left(N+\frac{W-M}{R}\right) \cdot \gamma+N \beta\right) \delta u\right|_{-\alpha} ^{\alpha}=0$

one obtains the following equilibrium equations for infinitesimal segment of the cross-section:

(1) the axial equilibrium

$$
\begin{aligned}
& -N^{\prime}+\frac{M^{\prime}}{R}-\frac{M \beta^{\prime \prime}}{R}+\left(Q_{\mathrm{x}}^{\prime}-N-\frac{q_{\mathrm{x}} R_{\mathrm{q}} x_{\mathrm{q}}}{R}\right) \beta+\left(Q_{\mathrm{x}}-\frac{M^{\prime}}{R}\right) \beta^{\prime} \\
& -N \varepsilon_{\mathrm{m}}^{\prime}+\left(Q_{\mathrm{x}}-N^{\prime}\right) \varepsilon_{\mathrm{m}}-N \gamma-q_{\mathrm{z}} R=0
\end{aligned}
$$

(2) the bending moment equilibrium

$-M^{\prime}-R Q_{\mathrm{x}}-\left(M \varepsilon_{\mathrm{m}}\right)^{\prime}-(W \kappa)^{\prime}-R Q_{\mathrm{x}} \varepsilon_{\mathrm{m}}-W \cdot \gamma+M \gamma+q_{\mathrm{x}} R_{\mathrm{q}} x_{\mathrm{q}} \beta=0$

(3) the radial balance:

$$
\begin{gathered}
N-Q_{\mathrm{x}}^{\prime}+N\left(\varepsilon_{\mathrm{m}}-\beta^{\prime}\right)+\frac{M \kappa+W \kappa}{R}-\left[\left(N+\frac{W-M}{R}\right) \gamma\right]^{\prime} \\
-\left(Q_{\mathrm{x}}+N^{\prime}\right) \beta-q_{\mathrm{x}} R=0
\end{gathered}
$$

Eqs. (27a-c) are derived without any simplification, they are used for buckling or nonlinear analysis with moderate displacements, the quantity $T$, defined in Eq. (21b) and appearing in Eqs. (20,23), disappears in Eqs. (27a,b,c). This is because the second order effects of different stresses cancel each other partly. If one neglects the effect of the transverse stress $\sigma_{\mathrm{x}}\left(\sigma_{\mathrm{y}}\right)$, some 
quantities will be left in the equilibrium equations and probably lead to unexpected solutions[4].

The boundary conditions are given in Table 1

Table 1

Boundary conditions

\begin{tabular}{cc}
\hline Displacement & The corresponding generalized force \\
\hline$\delta w$ & $\left(N-\frac{M}{R}\right)\left(1+\varepsilon_{\mathrm{m}}\right)+\frac{M}{R} \kappa-\left(Q_{\mathrm{x}}+\frac{T}{R}\right) \beta$ \\
\hline$\delta u$ & $Q_{\mathrm{x}}+\left(N+\frac{W-M}{R}\right) \cdot \gamma+N \beta=N \beta-\frac{M^{\prime}}{R}$ \\
\hline$\delta \theta / R$ & $-\frac{M \varepsilon_{\mathrm{m}}^{\prime}}{R}-\frac{(W \kappa)^{\prime}}{R}-\left(Q_{\mathrm{x}}+\frac{M^{\prime}}{R}\right) \varepsilon_{\mathrm{m}}+N \cdot \gamma$ \\
\hline & $M\left(1+\varepsilon_{\mathrm{m}}\right)+W \kappa+T \beta$ \\
\hline
\end{tabular}

From Eq. (27b) $\left(x_{q}=0\right.$ is introduced for simplicity $)$ :

$Q_{\mathrm{x}}=-\frac{M^{\prime}}{R}-Q_{\mathrm{x}} \varepsilon_{\mathrm{m}}-\frac{\left(M \varepsilon_{\mathrm{m}}+W \kappa\right)^{\prime}}{R}+\frac{(M-W) \gamma}{R}$

Substituting $Q_{\mathrm{x}}$ into Eq. (27c), one obtains

$N+\frac{M^{\prime \prime}}{R}+N\left(\varepsilon_{\mathrm{m}}-\beta^{\prime}\right)+\frac{M}{R}\left(\varepsilon_{\mathrm{m}}^{\prime \prime}+\kappa\right)+\left(\frac{2 M^{\prime}}{R}+Q_{\mathrm{x}}\right) \varepsilon_{\mathrm{m}}^{\prime}+\left(\frac{M^{\prime \prime}}{R}+Q_{\mathrm{x}}^{\prime}\right) \varepsilon_{\mathrm{m}}$

$-\left(Q_{x}+N^{\prime}\right) \beta+\frac{W \kappa}{R}+\left(\frac{W \kappa}{R}\right)^{\prime \prime}-(N \gamma)^{\prime}-R q_{x}=0$

\subsection{Differential equations for linear analysis}

Equilibrium equations for linear analysis are (Fig.4)

$Q_{\mathrm{x}}^{\prime}-N+q_{\mathrm{x}} R=0, \quad N^{\prime}+Q_{\mathrm{x}}+q_{\mathrm{z}} R=0, \quad M^{\prime}+Q_{\mathrm{x}} R=0$

$(30 \mathrm{a}, \mathrm{b}, \mathrm{c})$

Eliminating $Q_{\mathrm{x}}$ from the above equations

$N R+M^{\prime \prime}-q_{\mathrm{x}} R^{2}=0$

$N^{\prime} R-M^{\prime}+q_{\mathrm{z}} R^{2}=0$

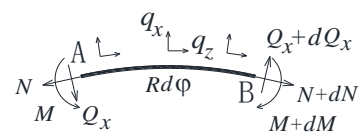

Fig.4 Balance of an infinitesimal segment

Substituting Eq. (8a) into Eqs. (16a, b, c), using Eq. (30c), the following relations are obtained for linear analysis

$$
\begin{aligned}
& N=E A \varepsilon_{\mathrm{m}}+\frac{E I \kappa}{R^{2}}=\frac{E A}{R}\left(w^{\prime}+u\right)+\frac{E I}{R^{3}}\left(u+R \theta^{\prime}\right) \\
& M=-\int_{A} \sigma_{\mathrm{z}} x \mathrm{~d} A=\frac{E I}{R^{2}}\left(R \theta^{\prime}+u\right), \quad Q_{\mathrm{x}}=G A_{\mathrm{s}}\left(\frac{u^{\prime}}{R}-\theta\right) \\
& W=\int_{A} \sigma_{\mathrm{z}} \frac{x^{2}}{r} \mathrm{~d} A=\int_{A} E\left(\varepsilon_{\mathrm{m}}-\frac{x}{r} \kappa\right) \frac{(r-R)^{2}}{r} \mathrm{~d} A \approx \frac{E I}{R^{2}}\left(w^{\prime}+u\right)
\end{aligned}
$$

Equilibrium differential equations in terms of displacements for linear analysis are:

$$
E A\left(w^{\prime \prime}+u^{\prime}\right)+q_{\mathrm{z}} R^{2}=0
$$

$E A\left(w^{\prime}+u\right)+\frac{E I}{R^{2}}\left(u+u^{\prime \prime}+R \theta^{\prime}+R \theta^{\prime \prime \prime}\right)-q_{\mathrm{x}} R^{2}=0$
$u^{\prime}=\frac{R}{1+\Phi}\left(\theta-\Phi \theta^{\prime \prime}\right)$

Differentiating Eq. (33b) once, and subtracting Eq.(33a) from it, one has

$\frac{E I}{R^{4}}\left(u^{\prime}+u^{\prime \prime \prime}+R \theta^{\prime \prime}+R \theta^{(4)}\right)=q_{\mathrm{x}}^{\prime}+q_{\mathrm{z}}$

Substituting Eq. (33c) into Eq. (34), the basic differential equation for linear analysis of Timoshenko's circular arch is obtained:

$\frac{E I}{(1+\Phi) R^{3}}\left(\theta^{(4)}+2 \theta^{\prime \prime}+\theta\right)=q_{\mathrm{x}}^{\prime}+q_{\mathrm{z}}$

Solving Eq. (35) for $\theta$, and then finding $u$ by Eq. (33c), and $w$ by Eq. (33a) or Eq. (33b), introducing boundary conditions, one may obtain the full linear solution of Timoshenko's circular arch.

\subsection{Differential Equations for Linearized Buckling Analysis}

Equations for linearized buckling analysis of circular arches are derived from Eqs. (27a,b,c), based on the static criterion for buckling.

An arch satisfying Eqs. $(27 \mathrm{a}, \mathrm{b}, \mathrm{c})$ is in a state of equilibrium. Introducing a small perturbation into the arch, letting it drift from this equilibrium state. When the perturbation is removed, if the arch is still in a state of equilibrium (still satisfying Eqs. (27a,b,c)), the equilibrium of the original system is neutral, the corresponding load is the critical load. The terms after perturbation are

$u+\dot{u}, w+\dot{w}, \beta+\dot{\beta}, \kappa+\dot{\kappa}, \varepsilon_{\mathrm{m}}+\dot{\varepsilon}_{\mathrm{m}}, \theta+\dot{\theta}, \gamma+\dot{\gamma}$

$q_{\mathrm{x}}+\dot{q}_{\mathrm{x}}, q_{\mathrm{z}}+\dot{q}_{\mathrm{z}}, N+\dot{N}, M+\dot{M}, Q_{\mathrm{x}}+\dot{Q}_{\mathrm{x}}, W+\dot{W}$

It is usually required that the loads are kept unchanged during the perturbation, here load increments are produced due to the change of loading direction as explained by Dinnik[7] and Simitses[3].

Substituting Eqs. (36a,b) into Eq. (27a) and Eq.(29), neglecting higher order terms, and noting that the system is in a state of equilibrium before the perturbation, the following equations for buckling analysis are obtained

$$
\begin{aligned}
& -\dot{N}^{\prime}+\frac{\dot{M}^{\prime}}{R}-N \dot{\varepsilon}_{\mathrm{m}}^{\prime}-\dot{N} \varepsilon_{\mathrm{m}}^{\prime}-\frac{M \dot{\beta}^{\prime \prime}}{R}-\frac{\dot{M} \beta^{\prime \prime}}{R}+\left(Q_{\mathrm{x}}^{\prime}-N\right) \dot{\beta}+\left(\dot{Q}_{\mathrm{x}}^{\prime}-\dot{N}\right) \beta \\
& +\left(Q_{\mathrm{x}}-\frac{M^{\prime}}{R}\right) \dot{\beta}^{\prime}+\left(\dot{Q}_{\mathrm{x}}-\frac{\dot{M}^{\prime}}{R}\right) \beta^{\prime}+\left(Q_{\mathrm{x}}-N^{\prime}\right) \dot{\varepsilon}_{\mathrm{m}}+\left(\dot{Q}_{\mathrm{x}}-\dot{N}^{\prime}\right) \varepsilon_{\mathrm{m}} \\
& -N \dot{\gamma}-\dot{N} \gamma-R \dot{q}_{\mathrm{z}}=0
\end{aligned}
$$

$\dot{N}+\frac{\dot{M}^{\prime \prime}}{R}+N\left(\dot{\varepsilon}_{\mathrm{m}}-\dot{\beta}^{\prime}\right)+\dot{N}\left(\varepsilon_{\mathrm{m}}-\beta^{\prime}\right)+\frac{M}{R}\left(\dot{\varepsilon}_{\mathrm{m}}^{\prime \prime}+\dot{\kappa}\right)+\frac{\dot{M}}{R}\left(\varepsilon_{\mathrm{m}}^{\prime \prime}+\kappa\right)+$ $\left(\frac{2 M^{\prime}}{R}+Q_{\mathrm{x}}\right) \dot{\varepsilon}_{\mathrm{m}}^{\prime}+\left(\frac{2 \dot{M}^{\prime}}{R}+\dot{Q}_{x}\right) \varepsilon_{\mathrm{m}}^{\prime}+\left(\frac{M^{\prime \prime}}{R}+Q_{\mathrm{x}}^{\prime}\right) \dot{\varepsilon}_{\mathrm{m}}+\left(\frac{\dot{M}^{\prime \prime}}{R}+\dot{Q}_{\mathrm{x}}^{\prime}\right) \varepsilon_{\mathrm{m}}$

$-\left(Q_{\mathrm{x}}+N^{\prime}\right) \dot{\beta}-\left(\dot{Q}_{\mathrm{x}}+\dot{N}^{\prime}\right) \beta+\frac{W \dot{\kappa}+\dot{W} \kappa}{R}+\left(\frac{\dot{W} \kappa+W \dot{\kappa}}{R}\right)^{\prime \prime}$

$$
-(N \dot{\gamma}+\dot{N} \gamma)^{\prime}-R \dot{q}_{\mathrm{x}}=0
$$

$$
\begin{aligned}
R \dot{Q}_{\mathrm{x}} & =-\dot{M}^{\prime}-\left(M \dot{\varepsilon}_{\mathrm{m}}+\dot{M} \varepsilon_{\mathrm{m}}\right)^{\prime}-(\dot{W} \kappa+W \dot{\kappa})^{\prime}-R \dot{Q}_{\mathrm{x}} \varepsilon_{\mathrm{m}}-R Q_{\mathrm{x}} \dot{\varepsilon}_{\mathrm{m}} \\
& +(M-W) \dot{\gamma}+(\dot{M}-\dot{W}) \gamma
\end{aligned}
$$

If one neglects the effect of the prebuckling deformation, then

$$
\begin{aligned}
& -\dot{N}^{\prime}+\frac{\dot{M}^{\prime}}{R}-N \dot{\varepsilon}_{\mathrm{m}}^{\prime}-\frac{M \dot{\beta}^{\prime \prime}}{R}+\left(Q_{\mathrm{x}}^{\prime}-N\right) \dot{\beta}+\left(Q_{\mathrm{x}}-\frac{M^{\prime}}{R}\right) \dot{\beta}^{\prime} \\
& +\left(Q_{\mathrm{x}}-N^{\prime}\right) \dot{\varepsilon}_{\mathrm{m}}-N \dot{\gamma}-R \dot{q}_{\mathrm{z}}=0 \\
& \dot{N}+\frac{\dot{M}^{\prime \prime}}{R}+N\left(\dot{\varepsilon}_{\mathrm{m}}-\dot{\beta}^{\prime}\right)+\frac{M}{R}\left(\dot{\varepsilon}_{\mathrm{m}}^{\prime \prime}+\dot{\kappa}\right)+\left(\frac{2 M^{\prime}}{R}+Q_{\mathrm{x}}\right) \dot{\varepsilon}_{\mathrm{m}}^{\prime}+\left(\frac{M^{\prime \prime}}{R}+Q_{\mathrm{x}}^{\prime}\right) \dot{\varepsilon}_{\mathrm{m}} \\
& -\left(Q_{\mathrm{x}}+N^{\prime}\right) \dot{\beta}+\frac{1}{R} W \dot{\kappa}+\frac{1}{R}(W \dot{\kappa})^{\prime \prime}-(N \dot{\gamma})^{\prime}-R \dot{q}_{\mathrm{x}}=0
\end{aligned}
$$


$R \dot{Q}_{\mathrm{x}}=-\dot{M}^{\prime}-\left(M \dot{\varepsilon}_{\mathrm{m}}\right)^{\prime}-(W \dot{\kappa})^{\prime}-R Q_{\mathrm{x}} \dot{\varepsilon}_{\mathrm{m}}-W \cdot \dot{\gamma}+M \dot{\gamma}$

If the prebuckling internal forces are computed by linear analysis, then these forces satisfy Eqs. (30a, b, c). Eqs. (38a, b, c) are further simplified into

$-\dot{N}^{\prime}+\frac{\dot{M}^{\prime}}{R}-N \dot{\varepsilon}_{\mathrm{m}}^{\prime}-\frac{M \dot{\beta}^{\prime \prime}}{R}-q_{\mathrm{x}} R \dot{\beta}-\frac{2 M^{\prime}}{R} \dot{\beta}^{\prime}+\left(Q_{\mathrm{x}}-N^{\prime}\right) \dot{\varepsilon}_{\mathrm{m}}-N \dot{\gamma}-R \dot{q}_{\mathrm{z}}=0(39 \mathrm{a})$

$\dot{N}+\frac{\dot{M}^{\prime \prime}}{R}+N\left(\dot{\varepsilon}_{\mathrm{m}}-\dot{\beta}^{\prime}\right)+\frac{M}{R}\left(\dot{\varepsilon}_{\mathrm{m}}^{\prime \prime}+\dot{\kappa}\right)+\frac{M^{\prime}}{R} \dot{\varepsilon}_{\mathrm{m}}^{\prime}+q_{\mathrm{z}} R \dot{\beta}+\frac{W \dot{\kappa}}{R}$

$+\left(\frac{W \dot{\kappa}}{R}\right)^{\prime \prime}-(N \dot{\gamma})^{\prime}-R \dot{q}_{\mathrm{x}}=0$

$R \dot{Q}_{\mathrm{x}}=-\dot{M}^{\prime}-M \dot{\varepsilon}_{\mathrm{m}}^{\prime}-(W \dot{\kappa})^{\prime}-W \cdot \dot{\gamma}+M \dot{\gamma}$

Because the incremental quantities are small, the increments of internal forces are given by

$\dot{N}=\frac{E A}{R}\left(\dot{w}^{\prime}+\dot{u}\right)+\frac{E I}{R^{3}}\left(R \dot{\theta}^{\prime}+\dot{u}\right), \quad \dot{M}=\frac{E I}{R^{2}}\left(R \dot{\theta}^{\prime}+\dot{u}\right)$

$\dot{Q}_{\mathrm{x}}=-\frac{E I}{R^{3}}\left(R \dot{\theta}^{\prime \prime}+\dot{u}^{\prime}\right)=G A_{\mathrm{s}}\left(\frac{\dot{u}^{\prime}}{R}-\dot{\theta}\right), \dot{W}=\frac{E I}{R^{2}}\left(\dot{w}^{\prime}+\dot{u}\right)$,

$\dot{\varepsilon}_{\mathrm{m}}=\frac{\dot{w}^{\prime}+\dot{u}}{R}, \quad \dot{\kappa}=\frac{\dot{u}}{R}+\dot{\theta}^{\prime}, \quad \dot{\beta}=\dot{\theta}-\frac{\dot{w}}{R}, \quad \dot{\gamma}=\frac{\dot{u}^{\prime}}{R}-\dot{\theta}, \quad \dot{\varepsilon}_{\mathrm{m}}-\dot{\kappa}=-\dot{\beta}^{\prime}$

Substituting Eqs. (42a-d) and Eqs. (43a-e) into Eqs. (41a-c), one has

$-\frac{E A}{R}\left(\dot{w}^{\prime \prime}+\dot{u}^{\prime}\right)-N \frac{\dot{w}^{\prime \prime}+\dot{u}^{\prime}}{R}-\frac{M}{R^{2}}\left(R \dot{\theta}^{\prime \prime}-\dot{w}^{\prime \prime}\right)-q_{\mathrm{x}}(R \dot{\theta}-\dot{w})-\frac{2 M^{\prime}}{R^{2}} \times$

$\left(R \dot{\theta}^{\prime}-\dot{w}^{\prime}\right)+\left(Q_{\mathrm{x}}-N^{\prime}\right) \frac{\dot{w}^{\prime}+\dot{u}}{R}-N\left(\frac{\dot{u}^{\prime}}{R}-\dot{\theta}\right)-R \dot{q}_{\mathrm{z}}=0$

$\frac{E A}{R}\left(\dot{w}^{\prime}+\dot{u}\right)+\frac{E I}{R^{3}}\left(R \dot{\theta}^{\prime \prime \prime}+R \dot{\theta}^{\prime}+\dot{u}^{\prime \prime}+\dot{u}\right)+\frac{N}{R}\left(2 \dot{w}^{\prime}+\dot{u}-R \dot{\theta}^{\prime}\right)$

$+\frac{M}{R^{2}}\left(\dot{w}^{\prime \prime \prime}+\dot{u}^{\prime \prime}+R \dot{\theta}^{\prime}+\dot{u}\right)+\frac{M^{\prime}}{R^{2}}\left(\dot{w}^{\prime \prime}+\dot{u}^{\prime}\right)+q_{\mathrm{z}}\left(R \dot{\theta}^{\prime}-\dot{w}\right)$

$+\frac{W\left(\dot{u}+R \dot{\theta}^{\prime}\right)}{R^{2}}+\left(\frac{W\left(\dot{u}+R \dot{\theta}^{\prime}\right)}{R^{2}}\right)^{\prime \prime}-\left[N\left(\frac{\dot{u}^{\prime}}{R}-\dot{\theta}\right)\right]^{\prime}-R \dot{q}_{\mathrm{x}}=0$

$$
\begin{aligned}
G A_{\mathrm{s}}\left(\dot{u}^{\prime}-R \dot{\theta}\right) & =-\frac{E I}{R^{2}}\left(R \dot{\theta}^{\prime \prime}+\dot{u}^{\prime}\right)-\frac{M}{R}\left(R \dot{\theta}+\dot{w}^{\prime \prime}\right) \\
& -W^{\prime}\left(\frac{\dot{u}}{R}+\dot{\theta}^{\prime}\right)-\frac{W}{R}\left(2 \dot{u}^{\prime}+R \dot{\theta}^{\prime \prime}-R \dot{\theta}\right)
\end{aligned}
$$

Eqs. (42a, b, c) are the linearized differential equations for buckling analysis of Timoshenko's arches, in which the effect of the prebuckling deformation has been neglected, but all prebuckling internal forces are retained.

\section{Buckling of rings under radial pressure}

\subsection{Prebuckling deformations}

The ring is acted on by the uniform radial pressure $p$ (along the centroid line), $q_{\mathrm{x}}=-p, q_{\mathrm{z}}=0$. The deformation is a uniform contraction, no shear deformation occurs. The linear solution is

$$
\begin{aligned}
& q_{\mathrm{x}}^{\mathrm{p}}=-p, \quad q_{\mathrm{z}}^{\mathrm{p}}=0, \quad N^{\mathrm{p}}=-p R, \quad w^{\mathrm{p}}=0, \quad \theta^{\mathrm{p}}=0, \quad \beta^{\mathrm{p}}=0, \quad Q_{\mathrm{x}}^{\mathrm{p}}=0 \\
& \gamma^{\mathrm{p}}=0, \chi=\frac{E I}{E A R^{2}}, \quad u^{\mathrm{p}}=-\frac{p R^{2}}{(1+\chi) E A}, \quad \varepsilon_{\mathrm{m}}^{\mathrm{p}}=\frac{u^{\mathrm{p}}}{R}, \quad \kappa^{\mathrm{p}}=\frac{u^{\mathrm{p}}}{R} \\
& M^{\mathrm{p}}=\frac{E I}{R^{2}} u^{\mathrm{p}}=-\frac{\chi}{1+\chi} p R^{2}, W^{\mathrm{p}} \approx \frac{E I}{R^{2}}\left(w^{\prime \mathrm{p}}+u^{\mathrm{p}}\right)=M^{\mathrm{p}}
\end{aligned}
$$

\subsection{Buckling Equations}

In this example Eqs. (42a, b, c) simplify to
$\frac{E A}{R}\left(\dot{w}^{\prime \prime}+\dot{u}^{\prime}\right)+N^{\mathrm{p}}\left(\frac{\dot{w}^{\prime \prime}+2 \dot{u}^{\prime}}{R}-\dot{\theta}\right)+\frac{M^{\mathrm{p}}}{R^{2}}\left(R \dot{\theta}^{\prime \prime}-\dot{w}^{\prime \prime}\right)+q_{\mathrm{x}}(R \dot{\theta}-\dot{w})=R \dot{q}_{\mathrm{z}}$

$\frac{E A}{R}\left(\dot{w}^{\prime}+\dot{u}\right)+\frac{E I}{R^{3}}\left(R \dot{\theta}^{\prime \prime \prime}+R \dot{\theta}^{\prime}+\dot{u}^{\prime \prime}+\dot{u}\right)+\frac{N^{p}}{R}\left(2 \dot{w}^{\prime}+\dot{u}-R \dot{\theta}^{\prime}\right)$

$+\frac{M^{p}}{R^{2}}\left(\dot{w}^{\prime \prime \prime}+R \dot{\theta}^{\prime \prime \prime}+2 \dot{u}^{\prime \prime}+2 R \dot{\theta}^{\prime}+2 \dot{u}\right)-N^{p}\left(\frac{\dot{u}^{\prime}}{R}-\dot{\theta}\right)^{\prime}-R \dot{q}_{\mathrm{x}}=0$

$G A_{\mathrm{s}}\left(\dot{u}^{\prime}-R \dot{\theta}\right)=-\frac{E I}{R^{2}}\left(R \dot{\theta}^{\prime \prime}+\dot{u}^{\prime}\right)-\frac{M^{p}}{R}\left(\dot{w}^{\prime \prime}+2 \dot{u}^{\prime}+R \dot{\theta}^{\prime \prime}\right)$

The effect of pre-buckling bending moment is further neglected, Eq. (44c) simplifies to

$\dot{u}^{\prime}=\frac{R}{1+\Phi}\left(\dot{\theta}-\Phi \dot{\theta}^{\prime \prime}\right)$

and Eqs. (44a, b) simplify to

$E A\left(\dot{w}^{\prime \prime}+\dot{u}^{\prime}\right)-p R\left(\dot{w}^{\prime \prime}-\dot{w}+2 \dot{u}^{\prime}\right)+R^{2} \dot{q}_{\mathrm{z}}=0$

$E A\left(\dot{w}^{\prime \prime}+\dot{u}^{\prime}\right)+\frac{E I}{(1+\Phi) R}\left(\dot{\theta}^{(4)}+2 \dot{\theta}^{\prime \prime}+\dot{\theta}\right)+N\left(2 \dot{w}^{\prime \prime}+\dot{u}^{\prime}-\dot{u}^{\prime \prime \prime}\right)-R^{2} \dot{q}_{\mathrm{x}}^{\prime}=0$

Considering three types of radial pressure (Fig.2):

Pressure 1: the direction of the pressure is unchanged during buckling, $\dot{q}_{x}=0, \dot{q}_{z}=0$;

Pressure 2: the pressure is directed toward the center of the arch, $\dot{q}_{x}=0$, $\dot{q}_{z}=-p \dot{w} / R$;

Pressure 3: the pressure is perpendicular to the tangent of the deformed centroid axis of the $\operatorname{arch}\left(\right.$ static water pressure), $\dot{q}_{\mathrm{x}}=0, \dot{q}_{\mathrm{z}}=-p\left(\dot{w}-\dot{u}^{\prime}\right) / R$

$\dot{q}_{\mathrm{x}}=\left\{\begin{array}{l}0 \\ 0 \\ 0\end{array}\right\}, \quad \dot{q}_{\mathrm{z}}=-\frac{p}{R}\left\{\begin{array}{c}0 \\ \dot{w} \\ \dot{w}-\dot{u}^{\prime}\end{array}\right\}$

Assuming that the buckling deformations are

$\dot{w}=B_{n} \cos n \phi, \quad \dot{\theta}=C_{n} \cos n \phi, \quad \dot{u}^{\prime}=\eta_{n} R C_{n} \cos n \phi$

$(47 \mathrm{a}, \mathrm{b}, \mathrm{c})$

where $\eta_{n}=\frac{1+n^{2} \Phi}{1+\Phi}$. Introduction of Eqs. $(47 \mathrm{a}, \mathrm{b}, \mathrm{c})$ into Eqs. $(45 \mathrm{~b}, \mathrm{c})$,

$\left[p R\left(n^{2}+1\right)-n^{2} E A\right] B_{n}+(E A-2 p R) \eta_{n} R C_{n}-p R\left\{\begin{array}{c}0 \\ B_{n} \\ B_{n}-\eta_{n} R C_{n}\end{array}\right\}=0$

$(2 p R-E A) n^{2} B_{n}+\left[E A \eta_{n}+\frac{\left(n^{2}-1\right)^{2} E I}{(1+\Phi) R^{2}}-p R\left(1+n^{2}\right) \eta_{n}\right] R C_{n}=0$

Let the determinant of coefficients of Eqs. (48a, b) vanish, one obtains the critical loads for three types of pressures $(n=2)$

$$
\begin{aligned}
& (p R)_{\mathrm{cr} 1}=\frac{4 E I}{(1+4 \Phi) R^{2}}\left(1-\frac{p R}{4(E A-p R)}\right) \\
& (p R)_{\mathrm{cr} 2}=\frac{4.5 E I}{(1+4 \Phi) R^{2}}\left(1-\frac{p R}{2 E A-p R}\right) \\
& (p R)_{\mathrm{cr} 3}=\frac{3 E I}{(1+4 \Phi) R^{2}}
\end{aligned}
$$

Eq.(49c) is the same as that by Smith and Simitses [8].

Considering that the shear stiffness and the axial stiffness may be in the same order of magnitudes, exact buckling loads may be found from Eq.(49a) and Eq.(49b) as follows 
$(p R)_{c r 1}=\frac{1}{2}\left[\frac{A R^{2}}{I}+\frac{5}{1+4 \Phi}-\sqrt{\left(\frac{A R^{2}}{I}+\frac{5}{1+4 \Phi}\right)^{2}-\frac{16}{1+4 \Phi} \cdot \frac{A R^{2}}{I}}\right] \frac{E I}{R^{2}}$

$=\eta_{1} \frac{4 E I}{(1+4 \Phi) R^{2}} \approx \frac{4 E I}{(1+4 \Phi) R^{2}}$

$(p R)_{c r 2}=\frac{1}{2}\left[\frac{2 A R^{2}}{I}+\frac{9}{1+4 \Phi}-\sqrt{\left(\frac{2 A R^{2}}{I}+\frac{9}{1+4 \Phi}\right)^{2}-\frac{36}{1+4 \Phi} \cdot \frac{A R^{2}}{I}}\right] \frac{E I}{R^{2}}$

$=\eta_{2} \frac{4.5 E I}{(1+4 \Phi) R^{2}} \approx \frac{4.5 E I}{(1+4 \Phi) R^{2}}$

Table 2 presents values of $\eta_{1}$ and $\eta_{2}$ for $R / i=5 \sim 100$ and $A / A_{s}=1.2 \sim 20$, both are close to 1.0

Table 2

Factors $\eta_{1}$ and $\eta_{2}$

\begin{tabular}{ccccccccc}
\hline \multirow{2}{*}{$\frac{A}{A_{s}}$} & \multicolumn{4}{c}{$\eta_{1}, R / i=$} \\
\cline { 2 - 9 } & 5 & 10 & 50 & 100 & 5 & 10 & 50 & 100 \\
\hline 1.2 & 0.971 & 0.991 & 1.00 & 1.00 & 0.940 & 0.980 & 0.999 & 1.000 \\
2 & 0.977 & 0.992 & 1.00 & 1.00 & 0.951 & 0.981 & 0.999 & 1.000 \\
5 & 0.987 & 0.993 & 1.00 & 1.00 & 0.971 & 0.985 & 0.999 & 1.000 \\
10 & 0.992 & 0.995 & 1.00 & 1.00 & 0.983 & 0.989 & 0.999 & 1.000 \\
20 & 0.996 & 0.997 & 1.00 & 1.00 & 0.990 & 0.993 & 0.999 & 1.000 \\
\hline
\end{tabular}

3.3 Solution under the assumption of inextensible arches

Inextensibility is frequently used to simplify solution of arch buckling. In the case of rings, if one adopts this assumption, $\dot{\varepsilon}_{\mathrm{m}}=0$, then

$\dot{w}^{\prime}=-\dot{u}$

Substituting Eq.(51) into Eq.(45c) leads to

$E I\left(\dot{\theta}^{(4)}+2 \dot{\theta}^{\prime \prime}+\dot{\theta}\right)+p R^{3}\left[\dot{\theta}+\dot{\theta}^{\prime \prime}-\Phi\left(\dot{\theta}^{\prime \prime}+\dot{\theta}^{(4)}\right)\right]=0$

from which one obtains $(p R)_{\mathrm{cr}}=(p R)_{\mathrm{cr} 3}$. Eq. $(45 \mathrm{~b})$ becomes

$p R\left(\dot{w}^{\prime \prime}+\dot{w}\right)-p R\left\{\begin{array}{c}0 \\ \dot{w} \\ \dot{w}+\dot{w}^{\prime \prime}\end{array}\right\}=0$

So, the axial equilibrium is satisfied only in the case of Pressure 3 (static water pressure). For Pressure 1 and 2, the axial equilibrium equation is not satisfied, so the inextensibility assumption should not be used for these two types of pressures, Eqs. (49a, b) indicate that the buckling loads are affected by the axial stiffness of the arch for both Pressure 1 and Pressure 2.

\section{Linear analysis of circular arches under radial pressure}

First we derive the prebuckling internal forces $N, M, Q_{\mathrm{x}}$, using Eq. (35) and Eqs. (33a, c). In the case of radial pressure, Eq. (35) is

$\theta^{(4)}+2 \theta^{\prime \prime}+\theta=\frac{\left(q_{\mathrm{x}}^{\prime}+q_{\mathrm{z}}\right)(1+\Phi) R^{3}}{E I}=0$

The general solution is

$$
\theta=C_{1} \cos \varphi+C_{2} \varphi \cos \varphi+C_{3} \sin \varphi+C_{4} \varphi \sin \varphi
$$

$u=R\left[C_{1} \sin \varphi+C_{2}(\varphi \sin \varphi+\cos \varphi)-C_{3} \cos \varphi+C_{4}(\sin \varphi-\varphi \cos \phi)\right]$

$-\frac{2 R \Phi}{1+\Phi}\left(C_{2} \cos \varphi+C_{4} \sin \varphi\right)+C_{5}$

Eq. (33b) becomes $w^{\prime}=\frac{q_{\mathrm{x}} R^{2}}{E A}-\frac{E I}{E A R^{2}} C_{5}-u$, integrating once:
$w=R\left[C_{1} \cos \varphi+C_{2}(\varphi \cos \varphi-2 \sin \varphi)+C_{3} \sin \varphi+C_{4}(\varphi \sin \varphi+2 \cos \varphi)\right]$

$$
+\frac{2 R \Phi}{1+\Phi}\left(C_{2} \sin \varphi-C_{4} \cos \varphi\right)-\left(1+\frac{1}{\rho^{2}}\right) C_{5} \varphi+C_{6}+\frac{q_{\mathrm{x}} R^{2}}{E A} \varphi
$$

where $\rho=R / i$. Because of the symmetry, $u$ is an even function, and $w$ is an odd function, $\phi$ starts at the arch top, $C_{1}=C_{4}=C_{6}=0$.Eq. (33a) becomes $E A\left(w^{\prime \prime}+u^{\prime}\right)=0$, so $E A\left(w^{\prime}+u\right) / R=$ constant, and Eq. (33b) is:

$u+u^{\prime \prime}+R \theta^{\prime}+R \theta^{\prime \prime \prime}=K=C_{5}$

$K=\frac{R^{3}}{E I_{\mathrm{y}}}\left(R q_{\mathrm{x}}-E A \frac{w^{\prime}+u}{R}\right)$

The boundary conditions are: $\theta= \pm \alpha: u=w=0, \theta^{\prime}=0,3$ constants are

$$
\begin{aligned}
& C_{2}=C_{20} \frac{q_{\mathrm{x}} R}{E A}, \quad C_{3}=C_{30} \frac{q_{\mathrm{x}} R}{E A}, \quad C_{5}=C_{50} \frac{q_{\mathrm{x}} R^{2}}{E A} \\
& C_{20}=-\frac{2 \alpha(1+\Phi) \cos \alpha}{2 \alpha\left[1+\Phi+2\left(1+1 / \rho^{2}\right) \cos ^{2} \alpha\right]-(3+\Phi) \sin 2 \alpha} \\
& C_{30}=(\alpha \tan \alpha-1) C_{20}, \quad C_{50}=-\frac{2 R \cos \alpha}{1+\Phi} C_{20}
\end{aligned}
$$

Thus, we obtain the linear exact solution of hinged shear-deformable arches under uniform radial pressure:

$$
\begin{aligned}
& u=\left[C_{20}\left(\varphi \sin \varphi+\frac{1-\Phi}{1+\Phi} \cos \varphi\right)-C_{30} \cos \varphi+C_{50}\right] \frac{q_{\mathrm{x}} R^{2}}{E A} \\
& w=\left[\varphi+\left(\phi \cos \phi-\frac{2}{1+\Phi} \sin \varphi\right) C_{20}+C_{30} \sin \varphi-\left(1+\frac{1}{\rho^{2}}\right) C_{50} \varphi\right] \frac{q_{\mathrm{x}} R^{2}}{E A}
\end{aligned}
$$

$\theta=\left(C_{20} \varphi \cos \varphi+C_{30} \sin \varphi\right) \frac{q_{\mathrm{x}} R}{E A}$

$u^{\prime}=\left(C_{20} \varphi \cos \varphi+C_{30} \sin \varphi+\frac{2 \Phi}{1+\Phi} C_{20} \sin \varphi\right) \frac{q_{\mathrm{x}} R^{2}}{E A}$

$\gamma=\frac{u^{\prime}}{R}-\theta=\frac{2 \Phi}{1+\Phi} \cdot \frac{q_{\mathrm{x}} R}{E A} \cdot C_{20} \sin \varphi$

The internal forces are

$N=q_{\mathrm{x}} R+\frac{2 E I}{(1+\Phi) R^{2}} C_{2} \cos \varphi=q_{\mathrm{x}} R\left(1+\frac{2}{(1+\Phi) \rho^{2}} C_{20} \cos \varphi\right)$

$M=\frac{1}{8} q_{\mathrm{x}} \alpha^{2} R^{2} \frac{2}{1+\Phi} \frac{8}{\alpha^{2} \rho^{2}} C_{20}(\cos \varphi-\cos \alpha)$

$Q_{\mathrm{x}}=G A_{\mathrm{s}} \gamma=-\frac{M^{\prime}}{R}=\frac{1}{2} q_{\mathrm{x}}(\alpha R)\left(\frac{4}{1+\Phi} \cdot \frac{1}{\alpha \rho^{2}}\right) C_{20} \sin \varphi$

$W \approx \frac{E I}{R^{2}}\left(w^{\prime}+u\right) \approx \frac{1}{\rho^{2}} q_{\mathrm{x}} R^{2}\left(1-\frac{1}{\rho^{2}} C_{50}\right)$

The linear solutions are illustrated in Fig.5, Fig.6 and Fig.7, where

$n=\frac{N}{p R}, m=\frac{M}{M_{0}}, M_{0}=\frac{q_{x}}{8}(\alpha R)^{2}, Q=\frac{Q_{\mathrm{x}}}{0.5 q_{\mathrm{x}} \alpha R}, U=u / \frac{q_{\mathrm{x}} R^{2}}{E A}, W=w / \frac{q_{\mathrm{x}} R^{2}}{E A}$

To highlight the effect of shear deformation, we take $\rho=10$ and $\Phi=0.0312$, $0.078,0.26,2.6$, corresponding to $A / A_{\mathrm{s}}=1.2,3,10,100$, respectively. It can be seen in Fig. 5, Fig. 6 and Fig. 7 that

(1) displacements become larger when shear deformation is included, but for the arch with $\alpha=60^{\circ}$, the increase is very small; and 


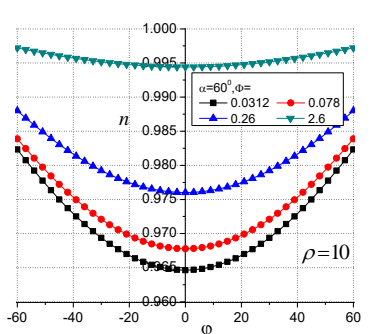

(a) axial force

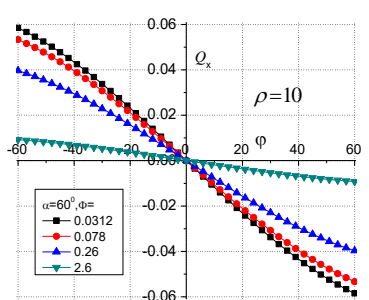

(c) shear force

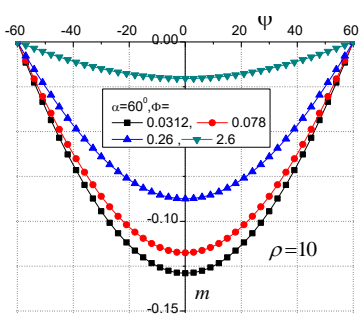

(b) bending moment

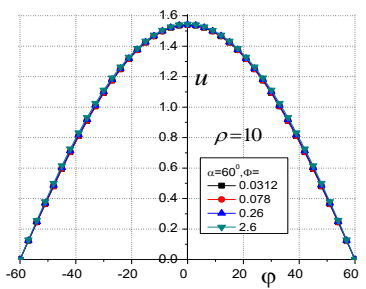

(d) radial displacement

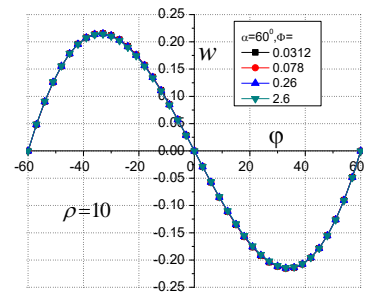

(e) circumferential displacement

Fig. 5 Linear solutions for $\rho=10, \alpha=60^{\circ}$

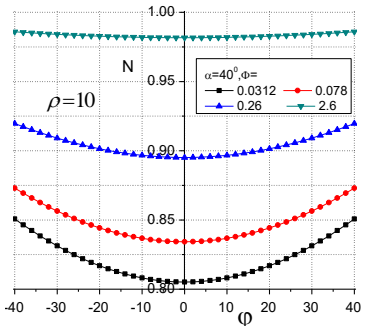

(a) axial force

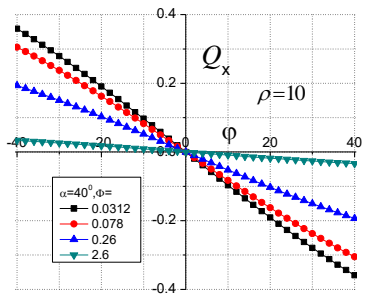

(c) shear force

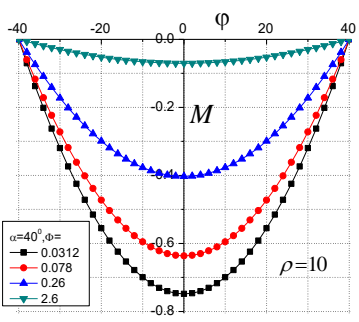

(b) bending moment

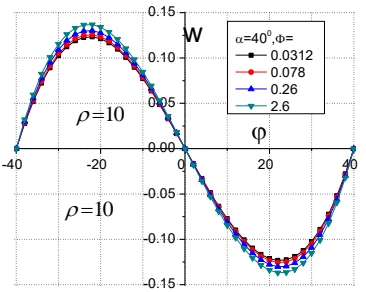

(d) radial displacement

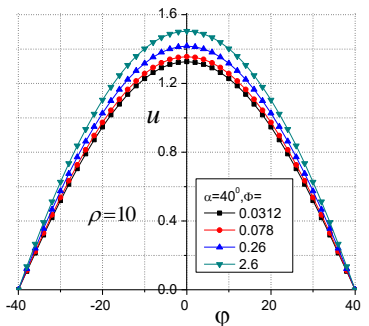

(e) circumferential displacement

Fig.6 Linear solution for $\rho=10, \alpha=40^{\circ}$

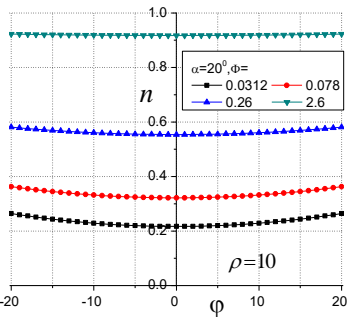

(a) axial force

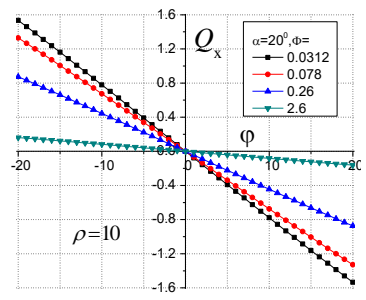

(c) shear force

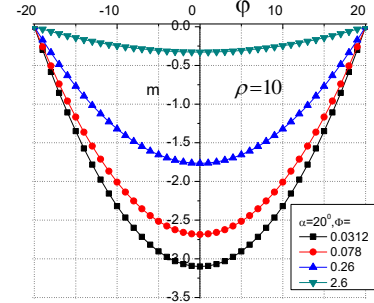

(b) bending moment

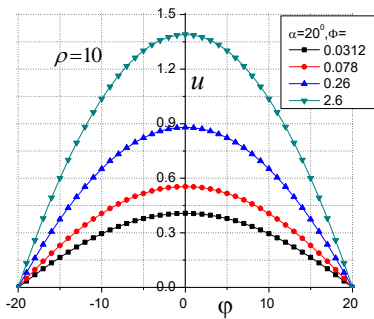

(d) radial displacement

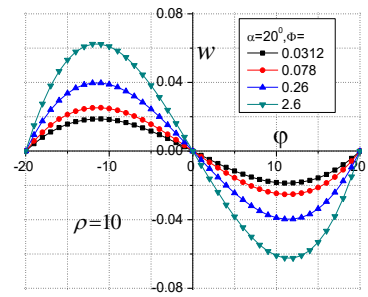

(e) circumferential displacement

Fig. 7 Linear solutions for $\rho=10, \alpha=20^{\circ}$

(2) the axial force is more uniform along the arch axis when shear deformation is included, and the bending moment and shear force are smaller. Such results are somewhat beyond expectation.

\section{Buckling of hinged circular arches}

This section addresses the buckling of shear-deformable hinged arches under three types of radial pressures

(1) Assuming that there is only the uniform axial force $N=q_{\mathrm{x}} R=-p R$. Assuming

$$
\dot{\theta}=D_{1} \cos \frac{\pi \varphi}{\alpha}, \quad \dot{w}=D_{2}\left(\cos \frac{\pi \varphi}{\alpha}+1\right)
$$

from Eq. (45a)

$$
\dot{u}^{\prime}=\frac{1+\Phi_{\mathrm{s}}}{1+\Phi} \cdot D_{1} R \cos \frac{\pi \varphi}{\alpha}, \quad \dot{u}=\frac{1+\Phi_{\mathrm{s}}}{1+\Phi} \cdot \frac{\alpha R}{\pi} D_{1} \sin \frac{\pi \varphi}{\alpha}
$$

Using Galerkin's method to Eqs. (45b, c), one obtains the critical loads for three types of radial pressures $p$.

For Pressure 1 (constant-direction):

$$
(p R)_{\mathrm{cr} 1}=\frac{\left(\pi^{2} / \alpha^{2}-1\right)^{2}}{\left(1+\Phi_{\mathrm{s}}\right)\left[\left(\frac{\pi^{2}}{\alpha^{2}}-1\right)^{2}+2\right]} \cdot \frac{E I}{R^{2}} \cdot \frac{E A-\left(1+3 \alpha^{2} / \pi^{2}\right) p R}{E A-p R+2 p R /\left(\frac{\pi^{2}}{\alpha^{2}}+3 \frac{\alpha^{2}}{\pi^{2}}-2\right)}
$$

from which

$$
(p R)_{\mathrm{cr} 1} \approx \frac{\psi_{1}}{1+\Phi_{\mathrm{s}}} \cdot \frac{\left(\pi^{2} / \alpha^{2}-1\right)^{2}}{\left(\pi^{2} / \alpha^{2}-1\right)^{2}+2} \cdot \frac{\pi^{2} E I}{(\alpha R)^{2}}
$$

$\psi_{1}=1-\frac{1}{1+0.2 \rho^{2}\left(1+\Phi_{s}\right)}$

For Pressure 2 (centered pressure):

$$
(p R)_{\mathrm{cr} 2}=\frac{\left(\pi^{2} / \alpha^{2}-1\right)^{2}}{\left(1+\Phi_{s}\right)\left(\pi^{2} / \alpha^{2}-2\right)} \cdot \frac{E I}{R^{2}} \cdot \frac{E A-p R}{E A-p R+p R /\left(\pi^{2} / \alpha^{2}-2\right)}
$$


From which

$(p R)_{\mathrm{cr} 2} \approx \frac{\psi_{2}}{1+\Phi_{s}} \cdot \frac{\left(\pi^{2} / \alpha^{2}-1\right)^{2}}{\left(\pi^{2} / \alpha^{2}-1\right)^{2}-1} \cdot \frac{\pi^{2} E I}{(\alpha R)^{2}}$

$\psi_{2}=1-\frac{1}{1+0.6 \rho^{2}\left(1+\Phi_{s}\right)}$

The parameters $\psi_{1}, \psi_{2}$ are solved for from Eq. (62) and Eq. (64), respectively, the expressions are complicated, exact values are compared with Eq. (63b) and Eq. (65b) in Fig.8, excellent agreements are found.

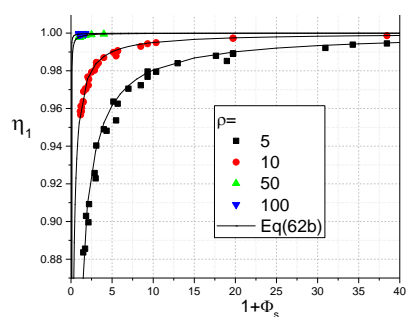

Pressure 1

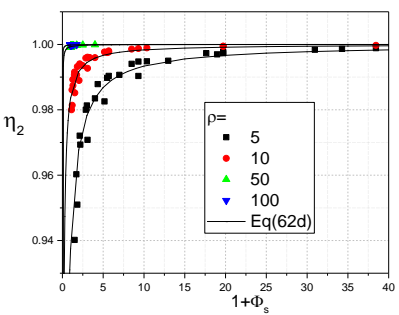

(b) Pressure 2
Fig.8 Coefficients $\psi_{1}, \psi_{2}$

and for Pressure 3 (water pressure):

$(p R)_{\mathrm{cr} 3}=\frac{1}{1+\Phi_{\mathrm{s}}} \cdot\left(\frac{\pi^{2}}{\alpha^{2}}-1\right) \frac{E I}{R^{2}}$

$(p R)_{\mathrm{cr} 3}$ is identical to Eq. (1) introduced by Dinnik [7].

When the shear stiffness of the cross-section is infinite, $\Phi_{\mathrm{s}}=0$, the critical loads are denoted by $(p R)_{\mathrm{cr}, \mathrm{f}}$, they are

$(p R)_{\mathrm{cr} 1, \mathrm{f}}=\frac{1}{1+0.2 \rho^{2}} \cdot \frac{\left(\pi^{2} / \alpha^{2}-1\right)^{2}}{\left(\pi^{2} / \alpha^{2}-1\right)^{2}+2} \cdot \frac{\pi^{2} E I}{\alpha^{2} R^{2}}$

$(p R)_{\mathrm{cr} 2, \mathrm{f}}=\frac{1}{1+0.6 \rho^{2}} \cdot \frac{\left(\pi^{2} / \alpha^{2}-1\right)^{2}}{\left(\pi^{2} / \alpha^{2}-1\right)^{2}-1} \cdot \frac{\pi^{2} E I}{\alpha^{2} R^{2}}$

$(p R)_{\mathrm{cr} 3, \mathrm{f}}=\left(1-\frac{\alpha^{2}}{\pi^{2}}\right) \cdot \frac{\pi^{2} E I}{\alpha^{2} R^{2}}$

On the other hand, if the bending stiffness is very large, and the shear stiffness is small, shear buckling of the arch will occur, the critical loads are denoted by $(p R)_{\mathrm{cr}, \mathrm{s}}$, they are obtained from Eqs. $(62,64,66)$ by setting $E I=\infty$

$(p R)_{\mathrm{cr} 1, \mathrm{~s}}=\left(1-\frac{2}{\left(\pi^{2} / \alpha^{2}-1\right)^{2}+2}\right) \frac{G A_{\mathrm{s}}}{\eta_{s 1}}, \quad \eta_{s 1}=1+5.2 \frac{\alpha^{2} G A_{s}}{\pi^{2} E A}$

$(p R)_{\mathrm{cr} 2, \mathrm{~s}}=\left(1+\frac{1}{\left(\pi^{2} / \alpha^{2}-1\right)^{2}-1}\right) \frac{G A_{\mathrm{s}}}{\eta_{s 2}}, \quad \eta_{s 2}=1+2.6 \frac{\alpha^{2} G A_{s}}{\pi^{2} E A}$

$(p R)_{\mathrm{cr} 3, \mathrm{~s}}=\left(1-\frac{\alpha^{2}}{\pi^{2}}\right) G A_{\mathrm{s}}$

So Eqs. $(63 a, 64 a, 66)$ may be expressed in form of interactive buckling

$\frac{1}{(p R)_{\mathrm{cr} i}}=\frac{1}{(p R)_{\mathrm{cri}, \mathrm{f}}}+\frac{1}{(p R)_{\mathrm{cr}, \mathrm{s}}} \quad i=1,2,3$

Eq. (69) is also valid for ring, and for ring

$$
\begin{aligned}
& (p R)_{\mathrm{cr} 1, \mathrm{f}}=\frac{\pi^{2} E I}{(0.5 \pi R)^{2}},(p R)_{\mathrm{cr} 2, \mathrm{f}}=\frac{9}{8} \frac{\pi^{2} E I}{(0.5 \pi R)^{2}},(p R)_{\mathrm{cr} 3, \mathrm{f}}=\frac{3}{4} \frac{\pi^{2} E I}{(0.5 \pi R)^{2}} \\
& (p R)_{\mathrm{cr} 1, \mathrm{~s}}=\frac{9}{11} G A_{\mathrm{s}}, \quad(p R)_{\mathrm{cr} 2, \mathrm{~s}}=\frac{9}{8} G A_{\mathrm{s}}, \quad(p R)_{\mathrm{cr} 3, \mathrm{~s}}=\frac{3}{4} G A_{\mathrm{s}}
\end{aligned}
$$

Assuming that the arches are uniformly compressed, and no other forces and deformations are considered. The buckling of such arches may be solved based on Eqs. (46a, b, c). Only water pressure (Pressure 3) is considered. In this particular pressure, the inextensible assumption is exactly satisfied, the differential equations for buckling of shear deformable circular arches under uniform compression are Eqs. (45a) and Eqs. $(51,52)$. The general solutions valid for any boundary conditions are

$$
\begin{aligned}
& \theta=C \sin \varphi+D \cos \varphi+\frac{1}{1-k^{2}}(A \sin k \varphi+B \cos k \varphi) \\
& u=-C R \cos \varphi+D R \sin \varphi+\frac{\left(1+\Phi k^{2}\right) R}{(1+\Phi)\left(1-k^{2}\right) k}(B \sin k \varphi-A \cos k \varphi)+F \\
& w=R(C \sin \varphi+D \cos \varphi)+\frac{\left(1+\Phi k^{2}\right) R(A \sin k \varphi+B \cos k \varphi)}{(1+\Phi)\left(1-k^{2}\right) k^{2}}-F \varphi+J \\
& M=\frac{E I}{R^{2}}\left[F-\frac{R}{(1+\Phi) k}(A \cos k \varphi-B \sin k \varphi)\right] \\
& Q_{x}=-\frac{E I}{(1+\Phi) R^{2}}(A \sin k \varphi+B \cos k \varphi)
\end{aligned}
$$

where $A, B, C, D, F, J$ are unknown constants, and

$k=\sqrt{\frac{1+p R^{3} / E I}{1-\Phi p R^{3} / E I}}$

\subsection{Fixed arch}

(1) Antisymmetrical buckling

In this case, $u$ is an odd function, $w$ and $\theta$ are even functions. The boundary conditions are: at $\varphi=\alpha: u=w=0, \theta=0$. The final critical equation is

$$
\tan k \alpha-\frac{(1+\Phi) k}{1+\Phi k^{2}} \tan \alpha=0
$$

The solutions are given in Table 3 in terms of buckling factors $K$, and the approximate expression is

$(p R)_{\mathrm{cr}}=\frac{K \pi^{2} E I}{(\alpha R)^{2}}=\frac{1}{\left(1+2.247 \Phi_{\mathrm{s}}\right)}\left(2.045-\frac{\alpha}{10 \pi}\right) \frac{\pi^{2} E I}{(\alpha R)^{2}}$

Table 3

Buckling factors of fixed arches: anti-symmetrical buckling

\begin{tabular}{cccccccc}
\hline & \multicolumn{7}{c}{$\Phi_{\mathrm{s}}$} \\
\cline { 2 - 7 }$\alpha$ & 0 & 0.05 & 0.1 & 0.2 & 0.3 & 0.4 & 0.5 \\
\hline $15^{0}$ & 2.0435 & 1.8370 & 1.6685 & 1.4100 & 1.2211 & 1.0770 & 0.9635 \\
$30^{\circ}$ & 2.0369 & 1.8310 & 1.6630 & 1.4052 & 1.2168 & 1.0731 & 0.9599 \\
$45^{0}$ & 2.0269 & 1.8220 & 1.6548 & 1.3981 & 1.2104 & 1.0673 & 0.9546 \\
$60^{\circ}$ & 2.0153 & 1.8116 & 1.6451 & 1.3899 & 1.2032 & 1.0608 & 0.9486 \\
$75^{0}$ & 2.0049 & 1.8021 & 1.6366 & 1.3826 & 1.1969 & 1.0550 & 0.9434 \\
$90^{\circ}$ & 2.0000 & 1.7978 & 1.6328 & 1.3793 & 1.1940 & 1.0528 & 0.9413 \\
\hline
\end{tabular}

(2) Symmetrical buckling: The critical equation is

$\frac{\left(1+\Phi k^{2}\right)}{(1+\Phi) k^{2}}(\alpha k \cot k \alpha-1)=\alpha \cot \alpha-1$

The solutions are given in Table 4 in terms of buckling factors $K$, and the approximate expression is

$(p R)_{\mathrm{cr}}=\frac{K \pi^{2} E I}{(\alpha R)^{2}}=\frac{1}{\left(1+3.96 \Phi_{\mathrm{s}}\right)}\left(3.366-\frac{9 \alpha^{2}}{16 \pi^{2}}\right) \frac{\pi^{2} E I}{(\alpha R)^{2}}$ 
Table 4

Buckling factors of fixed arches: symmetrical buckling

\begin{tabular}{cccccccc}
\hline & \multicolumn{7}{c}{$\Phi_{\mathrm{s}}$} \\
\cline { 2 - 7 }$\alpha$ & 0 & 0.05 & 0.1 & 0.2 & 0.3 & 0.4 & 0.5 \\
\hline $15^{0}$ & 3.3615 & 2.8059 & 2.4107 & 1.8861 & 1.5537 & 1.3239 & 1.1553 \\
$30^{0}$ & 3.3491 & 2.7952 & 2.4011 & 1.8780 & 1.5464 & 1.3173 & 1.1492 \\
$45^{0}$ & 3.3288 & 2.7777 & 2.3855 & 1.8648 & 1.5347 & 1.3066 & 1.1393 \\
$60^{0}$ & 3.3009 & 2.7537 & 2.3641 & 1.8468 & 1.5187 & 1.2920 & 1.1257 \\
$75^{0}$ & 3.2663 & 2.7240 & 2.3377 & 1.8245 & 1.4990 & 1.2741 & 1.1092 \\
$90^{\circ}$ & 3.2258 & 2.6895 & 2.3073 & 1.7990 & 1.4765 & 1.2535 & 1.0903 \\
\hline
\end{tabular}

6.2 Symmetrical buckling of hinged arches

The critical equation is

$\frac{\left(1+\Phi k^{2}\right) \tan k \alpha-k \alpha}{k^{3}}=(1+\Phi) \tan \alpha-\alpha$

The buckling factors $K$ are listed in Table 5, and the approximate expression is

$(p R)_{\mathrm{cr}}=K \frac{\pi^{2} E I}{(\alpha R)^{2}}=\frac{1}{\left(1+2.245 \Phi_{\mathrm{s}}\right)}\left(2.225-\frac{8 \alpha^{2}}{9 \pi^{2}}\right) \frac{\pi^{2} E I}{(\alpha R)^{2}}$

Table 5

Buckling factors $K$ of hinged arches: symmetrical buckling

\begin{tabular}{cccccccc}
\hline & \multicolumn{7}{c}{$\Phi_{\mathrm{s}}$} \\
\cline { 2 - 7 }$\alpha$ & 0 & 0.05 & 0.1 & 0.2 & 0.3 & 0.4 & 0.5 \\
\hline $15^{0}$ & 2.2192 & 1.9951 & 1.8121 & 1.5313 & 1.3259 & 1.1691 & 1.0455 \\
$30^{0}$ & 2.2002 & 1.9779 & 1.7965 & 1.5180 & 1.3144 & 1.1589 & 1.0364 \\
$45^{0}$ & 2.1686 & 1.9495 & 1.7706 & 1.4961 & 1.2953 & 1.1421 & 1.0213 \\
$60^{0}$ & 2.1246 & 1.9098 & 1.7344 & 1.4654 & 1.2687 & 1.1186 & 1.0002 \\
$75^{0}$ & 2.0682 & 1.8592 & 1.6884 & 1.4264 & 1.2349 & 1.0887 & 0.9734 \\
$90^{0}$ & 2.0000 & 1.7978 & 1.6328 & 1.3793 & 1.1940 & 1.0528 & 0.9413 \\
\hline
\end{tabular}

\subsection{Symmetrical buckling of arches with three hinges}

The anti-symmetrical buckling load is the same as the antisymmetrical buckling of hinged arches. In the case of symmetrical buckling, 5 boundary conditions are

$\varphi=\alpha: u=w=0, M=0 ; \quad \varphi=0: \quad w=0, M=0$

The sixth boundary condition comes from Table 1 . Because $u \neq 0$ at the apex of the arch, the force must vanish, $Q_{\mathrm{x}}-p \cdot\left(u^{\prime}-w\right)=0$. Introducing Eqs. (72a-e) into these boundary conditions, it obtains

$$
\frac{\left(1+\Phi k^{2}\right) \tan 0.5 k \alpha-0.5 k \alpha}{(0.5 k \alpha)^{3}}=\frac{4[(1+\Phi) \tan \alpha-\alpha]}{\alpha^{3}}
$$

The buckling factors $K$ are given in Table 6 , and the approximate expression is

$$
(p R)_{\mathrm{cr}}=K \frac{\pi^{2} E I}{(\alpha R)^{2}}=\frac{3}{4} \frac{\pi^{2} E I}{\left(1+\Phi_{\mathrm{s}}\right)(\alpha R)^{2}}
$$

Table 6

Buckling factors of arches with 3 hinges: symmetrical buckling

\begin{tabular}{cccccccc}
\hline & \multicolumn{7}{c}{$\Phi_{\mathrm{s}}$} \\
\cline { 2 - 8 }$\alpha$ & 0 & 0.05 & 0.1 & 0.2 & 0.3 & 0.4 & 0.5 \\
\hline $15^{0}$ & 0.7525 & 0.7166 & 0.6840 & 0.6270 & 0.5787 & 0.5373 & 0.5015 \\
$30^{0}$ & 0.7521 & 0.7163 & 0.6837 & 0.6267 & 0.5784 & 0.5371 & 0.5013 \\
$45^{0}$ & 0.7516 & 0.7158 & 0.6832 & 0.6263 & 0.5780 & 0.5367 & 0.5009 \\
$60^{0}$ & 0.7509 & 0.7151 & 0.6826 & 0.6257 & 0.5776 & 0.5363 & 0.5006 \\
$75^{0}$ & 0.7503 & 0.7146 & 0.6821 & 0.6252 & 0.5771 & 0.5359 & 0.5002 \\
$90^{0}$ & 0.7500 & 0.7143 & 0.6818 & 0.6250 & 0.5770 & 0.5358 & 0.5000 \\
\hline
\end{tabular}

6.4 Fixed arches with a hinge at the apex of the arch

The buckling load of anti-symmetrical buckling is the same as that of fixed arches. The critical equation for symmetrical buckling is

$$
\begin{aligned}
& \left\{\left(1-k^{2}\right) \cos k \alpha+\left[\frac{\left(1+\Phi k^{2}\right)}{\left(1-k^{2}\right)} \cos k \alpha+k \alpha \sin k \alpha-2\right]\left(1+\Phi k^{2}\right)\right\} \frac{\tan \alpha}{(1+\Phi) k} \\
& +k \alpha \cos k \alpha-\frac{\left(1+\Phi k^{2}\right)}{\left(1-k^{2}\right)} \sin k \alpha=0
\end{aligned}
$$

The buckling factors $K$ are presented in Table 7, and the approximate expression is

$$
(p R)_{\mathrm{cr}}=K \frac{\pi^{2} E I}{(\alpha R)^{2}}=\frac{1}{\left(1+2.245 \Phi_{\mathrm{s}}\right)}\left(1.112+\frac{\alpha^{2}}{6 \pi^{2}}\right) \frac{\pi^{2} E I}{(\alpha R)^{2}}
$$

Table 7

Buckling factors of arches with one hinge: symmetrical buckling

\begin{tabular}{cccccccc}
\hline & \multicolumn{7}{c}{$\Phi_{\mathrm{s}}$} \\
\cline { 2 - 8 }$\alpha$ & 0 & 0.05 & 0.1 & 0.2 & 0.3 & 0.4 & 0.5 \\
\hline $15^{0}$ & 1.114 & 0.999 & 0.907 & 0.770 & 0.672 & 0.599 & 0.543 \\
$30^{0}$ & 1.117 & 1.002 & 0.910 & 0.772 & 0.674 & 0.601 & 0.544 \\
$45^{0}$ & 1.122 & 1.007 & 0.914 & 0.776 & 0.677 & 0.604 & 0.546 \\
$60^{0}$ & 1.129 & 1.014 & 0.921 & 0.782 & 0.682 & 0.608 & 0.549 \\
$75^{0}$ & 1.140 & 1.024 & 0.930 & 0.790 & 0.689 & 0.613 & 0.554 \\
$90^{0}$ & 1.154 & 1.037 & 0.943 & 0.800 & 0.698 & 0.621 & 0.561 \\
\hline
\end{tabular}

6.5 Comparison between proposed formulas and FE buckling analysis

Table 8 gives some comparison between proposed formulas and FE buckling analysis for the arch $\alpha=45^{\circ}, R=13.56 \mathrm{~m}$ under various boundary conditions, the cross-section is $H 500 \times 300 \times 10 \times 16$, and $\Phi_{\mathrm{s}}=0.2669$. Comparison shows that the proposed formulas are on the safe side. The largest difference is $5.9 \%$ occurring in fixed arched for which the actual uniformity of the axial force in the arch is slightly larger.

Table 8

Comparison of $N_{\mathrm{cr}} / R$ between proposed formulas and FE buckling analysis

\begin{tabular}{ccccccc}
\hline & \multicolumn{2}{c}{ Anti-symmetrical buckling } & \multicolumn{3}{c}{ Symmetrical buckling } \\
\cline { 2 - 7 } & FEM & Eqn & FEM/Eqn & FEM & Eqn & FEM/Eqn \\
\hline Hinged arch & 597.6 & 594.3 & 0.995 & 1073.2 & 1031.5 & 0.961 \\
Fixed arch & 1040.6 & 979.8 & 0.942 & 1312.2 & 1257.5 & 0.958 \\
Three hinges & 594.7 & 591.3 & 0.994 & 458.9 & 447.4 & 0.975 \\
One hinge & 1028.9 & 968.1 & 0.941 & 549.9 & 538.1 & 0.979 \\
\hline
\end{tabular}

\section{Conclusions}

This paper derived a new theory for nonlinear and buckling analysis of shear-deformable circular arches. The derivation is based on the well-accepted Timoshenko's assumption on the deformation of the cross-section, the Green strains are used. Based on the virtual work principle, the paper derived a new set of equilibrium equations for buckling analysis and nonlinear analysis with moderate displacements considering the shear deformation of the cross-section of the arches. Compared with others, the present paper included not only the nonlinear effect of longitudinal and shear stresses in the arch, but also the nonlinear effect of the transverse normal stresses in the arch. This was included because the authors believed that the second order effects of all the stresses necessary to keep equilibrium of the plate or shell element will cancel each other partly, and neglect of any one of them will probably lead to incorrect solutions.

Linearization was carried out to obtain a set of differential equations suitable for buckling analysis. These equations are then used to solve the buckling of shear-deformable circular arches and rings under radial pressures to compare the various results appeared in the literature.

Linear analysis is carried out on hinged arches under uniform radial pressure to see the changes of displacements and internal forces after the shear deformation is included. It is found that the displacements become larger when shear deformation is included, but for the arch with $\alpha=60^{\circ}$, the increase is very 
small. The axial force is more uniform along the arch when shear deformation is considered, and the bending moment and shear force are smaller.

The buckling factors for symmetrical and anti-symmetrical buckling under

\section{References}

[1] Timoshenko, S. P, Gere, J. M., Theory of elastic stability, McGrawHill-Kogakusha Ltd, Tokyo, 109,1961.

[2] Pi Y.L., Bradford M.A. and Uy B., "In-plane stability of arches", International Journal of Solids and Structures, 39(1), 105-125, 2002.

[3] Simitses G.J., An introduction to the elastic stability of structures, Prentice Hall Inc, Englewood Cliffs, 1976.

[4] Tong G.S., Pi Y.L., Bradford M.A. and Tin-Loi F., "In-plane nonlinear buckling analysis of deep circular arches incorporating transverse stresses", Journal of engineering mechanics, 134(5), 362-373, 2008.

[5] Friedman Z. and Kosmatka Z.B., "An Accurate two-node finite element for shear deformable curved beams", International Journal for Numerical Methods in Engineering, 41, 473-498, 1998.

[6] Huang C.S, Nieh K.Y. and Yang M.C., "In-plane free vibration and stability of loaded and shear-deformable circular arches", International Journal of Solids and Structures, 40, 5865-5886, 2003

[7] Dinnik A.N., "Stability of arches", OGIS, Gostechizdat (in Russian, Chinese translation in 1958), 1946

[8] Smith C.V. and Simitses G.J., "Effect of shear and load behavior on ring stability", Journal of various boundary conditions are tabulated, and approximate formulas for the critical loads are proposed. the Engineering Mechanics Division, 95(3), 559-570, 1969.

[9] Guo Y.L. and Huang L., "Design theory and method for in-plane ultimate strength of arche with web openings", Journal of Building Structures, 3, 003, 2007.

[10] Dou C., Guo Y.F., Jiang Z.Q., Gao W. and Pi Y.L., "In-plane buckling and design of steel tubular truss arches", Thin-Walled Structures, 130, 613-621, 2018

[11] Guo Y.L., Guo Y.F. and Dou C., "In-plane buckling and design of two-hinged steel tube circular truss-arches under pure compression", Journal of Building Structures, 31(8), 45-53, 2010.

[12] Guo Y.L., Chen H., Pi Y.L. and Bradford M.A., "In-plane strength of steel arches with a sinusoidal corrugated web under a full-span uniform vertical load: Experimental an numerical investigations", Engineering Structures, 110, 105-115, 2016.

[13] Attard M.M., Zhu J.B. and Kellermann D.C.. "In-plane buckling of circular arches and ring with shear deformations", Archive of Applied Mechanics, 83(8), 1145-1169, 2013.

[14] Zhu J.B., Attard M.M. and Kellermann D.C., "In-plane nonlinear buckling of circular arches including shear deformation", Archive of Applied Mechanics, 84, 1841-1860, 2014.

[15] Attard M.M., Zhu J.B. and Kellermann D.C., "In-plane buckling of prismatic funicular arches with shear deformations", Archive of Applied Mechanics, 84, 693-713, 2014.

[16] Washizu K., Variational methods in elasticity and plasticity, Pergamon press, 1975. 